

\section{ABSTRACT}

12 Molecular-dynamics simulations are used for predictions of the glass transition temperatures for a test

13 set of 5 aprotic ionic liquids. Glass transitions are localized with the trend-shift method analyzing

14 volumetric and transport properties of bulk amorphous phases. Classical non-polarizable all-atom

15 OPLS force-field model developed by Canongia Lopes and Pádua (CL\&P) is employed as the starting

16 level of theory for all calculations. Alternative approaches of charge scaling and Drude oscillator

17 model, accounting for atomic polarizability either implicitly or explicitly, respectively, are used to

18 investigate the sensitivity of the glass transition temperatures to induction effects. The former non-

19 polarizable model overestimates the glass transition temperature by tens of Kelvins (37 K in average).

20 The charge scaling technique yields a significant improvement, and the best estimations were achieved

21 using polarizable simulations with the Drude model, which yielded an average deviation of $11 \mathrm{~K}$.

22 Although the volumetric data usually exhibit a lesser trend shift upon vitrification, their lower statistical

23 uncertainty enables to predict the glass transition temperature with a lower uncertainty than the ionic

24 self-diffusivities, the temperature dependence of which is usually more scattered. Additional analyses

25 of the simulated data were also performed, revealing that the Drude model predicts lower densities for

26 most sub-cooled liquids, but higher densities for the glasses than the original CL\&P, and that the Drude

27 model also invokes some longer-range organization of the sub-cooled liquid, greatly impacting the

28 temperature trend of ionic self-diffusivities in the low-temperature region. 


\section{INTRODUCTION}

31 Knowledge of the melting temperature is a key prerequisite for each liquid material to become

32 technologically applicable. However, numerous ionic liquids (ILs) have been reported not to crystallize

33 upon cooling or to crystallize with difficulties and irreproducibility. ${ }^{1}$ Large cohesive forces among the

34 individual ions render the fluid highly viscous, which impedes the formation of a regular crystal lattice

35 upon cooling. Instead, ILs often exhibit massive super-cooling of the liquid phase which undergoes a

36 phase transition to an amorphous glass-like solid state at temperatures far below the equilibrium

37 melting temperature. Knowledge of the respective glass transition temperature $\left(T_{\mathrm{g}}\right)$ thus becomes of

38 utmost importance in the case of ILs.

39 All phase transitions are relatively slow processes, the time scale of which can hardly be sampled

40 directly in all atom molecular-dynamics (MD) simulations covering at most dozens of nanoseconds for

41 condensed-phase samples. ${ }^{2}$ However, a phase transition is typically accompanied with a discontinuity

42 in the temperature trend (or at least a trend shift) of the properties of the given material, such as its heat

43 capacity, density, or diffusivity. Localization of the trend shift from simulated data then enables

44 prediction of $T_{\mathrm{g}}$ between a solid amorphous glassy state and a liquid. Analyzing the temperature

45 dependence of density or diffusivity enables thus in principle to derive the temperature of the phase

46 transition between the liquid and glassy states. ${ }^{3-5}$

47 While experimental determinations of the heat capacity usually point to a possible phase transition, its

48 high sensitivity to the computational noise makes it rather an unsuitable property to be observed

49 computationally in this case. On the other hand, densities, which are closely related to molar volumes,

50 have been reported to exhibit distinct trend shifts at the temperature of the glass transition for many

51 macromolecular and low-molecular materials, ${ }^{6-7}$ including ionic liquids. ${ }^{1,3,8}$ Despite the principal

52 simplicity of extraction of the densities from molecular simulations, concave temperature-dependence

53 of densities of the amorphous phases of ionic liquids (IL) can be rather gradual throughout the phase 
54 transition region, which complicates precise localization of $T_{\mathrm{g}}$ and leads to a larger statistical 55 uncertainty of such determination of $T_{\mathrm{g}}$. At the level of the non-polarizable force field, we illustrated 56 that the computational uncertainty of $T_{\mathrm{g}}$ reaches $20 \mathrm{~K}$ for alkylimidazolium $\mathrm{ILs},{ }^{1}$ but over $60 \mathrm{~K}$ for 57 various pharmaceutic ingredients. ${ }^{9}$

58 Last but not least, the diffusivity of liquids and solids differs by several orders of magnitude by 59 definition. Unfortunately, widespread non-polarizable force fields for IL tend to underestimate the 60 diffusivities of IL by 1-2 orders of magnitude, ${ }^{10-12}$ rendering the trend shift analysis of such 61 miscalculated data rather problematic. This phenomenon clearly arises due to large electrostatic 62 cohesive forces that arrange the ions into virtual cages that hinder other particles to move through. ${ }^{13}$ 63 Although there are also fixed-charge force fields ${ }^{14-15}$ (usually using united atoms) that yield fairly 64 accurate diffusivities with non-scaled atomic charges, a charge-scaling approach is nowadays 65 commonly used to fix this issue of all-atom force fields, aiming to account for the missing polarizability 66 of ions or charge transfer effects at least partially. ${ }^{16-17}$ When all atomic charges are scaled by an 67 empirical factor lesser than unity (around 0.8), ${ }^{18}$ the resulting diffusivity of ILs matches experimental 68 data appreciably better. Clearly, there are serious concerns about all the energetic aspects and 69 credibility of the interaction energies and structural features resulting from this charge-scaling 70 approach. ${ }^{19-20}$

71 An efficient way of modeling the atomic polarizability represents the Drude oscillator model. ${ }^{21}$ It 72 consists in replacing all polarizable atoms by two fictive particles connected by a harmonic oscillator 73 bond - a heavy atomic core and a light electron cloud whose masses and charges summed together 74 give the values of the original atom. In this way, the charge distribution of a molecule can fluctuate 75 over time, which corresponds to formation of induced dipoles that respond to the outer electric field of 76 surrounding ions. The Drude model has existed for some time, but its implementation in important MD 77 codes, such as Lammps, was achieved only recently. ${ }^{22}$ While there is an obvious computational way 
78 how to parametrize the atomic polarizabilities from quantum chemical calculations, ${ }^{23-24}$ optimum setup

79 of the parameters of the fictive Drude electrons (mass, charge, force constant, damping of interactions

80 of close Drude pairs) is still a subject of a scientific debate in the literature. ${ }^{20,25}$ These facts, together

81 with an appreciably higher computational cost of polarizable MD in general, imped using polarizable

82 simulations to study ILs-related phenomena, especially their phase transitions.

83 This work presents a detailed benchmark of MD-powered predictions of the glass transition

84 temperatures of five synthetic aprotic ILs. The performance of the popular non-polarizable all-atom

85 CL\&P force field ${ }^{26}$ and its polarizable CL\&P-D successor ${ }^{20}$ is compared. Moreover, within the non-

86 polarizable CL\&P FF, we also tested the approach with atomic charges scaled down by an empirical

87 factor of 0.8 . Thus, the total of three FF models were investigated for their performance in predicting

$88 T_{\mathrm{g}}$. The 5 ILs given, consisting of various archetypal cations and anions, were selected upon availability

89 of the experimental data on $T_{\mathrm{g}}$. The list of the considered ILs together with their chemical identifiers is

90 provided in Table 1.

91

92 TABLE 1

93 Overview of the ILs included in this study and their chemical identifiers.

\begin{tabular}{lcc}
\hline Ionic liquid & Formula & CAS RN \\
\hline 1-butyl-3-methylimidazolium bistriflimide & {$[$ bmIm] [NTf 2$]$} & $174899-83-3$ \\
1-butyl-3-methylimidazolium triflate & {$[$ bmIm][OTf $]$} & $174899-66-2$ \\
1-butyl-3-methylimidazolium tosylate & {$[$ bmIm][OTs $]$} & $410522-18-8$ \\
1-butylpyridinium bistriflimide & {$\left[\right.$ bPyri] $\left[\mathrm{NTf}_{2}\right]$} & $187863-42-9$ \\
1-butyl-1-methylpyrrolidinium bistriflimide & {$\left[{\text { bmPyrr }]\left[\mathrm{NTf}_{2}\right]}^{223437-11-4}\right.$} \\
\hline
\end{tabular}

94 


\subsection{Simulations}

97 The three FF models applied to predict $T_{\mathrm{g}}$ are termed throughout this work as follows:

- CL\&P: the original CL\&P model (non-polarizable);

- CL\&P-scq: the CL\&P model with all atomic charges $(q)$ scaled by a factor of $0.8\left(q^{\text {CL\&P-scq }}=\right.$

$$
\left.0.8 q^{\mathrm{CL \& P}}\right) \text { (non-polarizable); }
$$

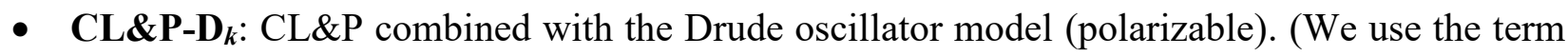
CL\&P-D $k$ in consistency with our recent paper, ${ }^{25}$ although the term "CL\&Pol" can also be found for this FF in the literature. ${ }^{27}$ )

104 To make the comparative study presented in this work as fair as possible, we used the same computational details regarding the MD simulations performed for all three FF models, at least where possible. The employed and thoroughly validated computational setup is based on our previous extensive computational studies addressing thermodynamic properties of IL using both nonpolarizable $\mathrm{e}^{1,28-29}$ and polarizable ${ }^{25}$ models.

110 configurations of the liquid-phase simulation boxes were generated by the PACKMOL program ${ }^{32}$ and

111 contained 300 to 480 IL pairs, depending on the number of atoms in the given IL pair. First, the

112 simulation box from PACKMOL for each IL was pre-equilibrated for $10 \mathrm{~ns}$ at an elevated temperature

113 of $400 \mathrm{~K}$. Then, copies of the pre-equilibrated boxes were made and equilibrated for another $5 \mathrm{~ns}$ at

114 different temperatures ranging from 100 to $400 \mathrm{~K}$, followed by a production period of $10 \mathrm{~ns}$ at each

115 temperature, sampling the system and recording the studied properties (mainly density and mean-

116 square displacement) every $1000 \mathrm{fs}$. The velocity Verlet integrator with a time step value of $1 \mathrm{fs}$ was

117 used in all cases. The Nosé-Hoover $(\mathrm{NH})$ thermostat and barostat were applied to control the 
118 simulation temperature and pressure (always $0.1 \mathrm{MPa}$ ) in $N P T$ ensembles, with update frequencies of

119100 and $1000 \mathrm{fs}$, respectively. Regarding CL\&P-D $k$, we applied the temperature-grouped dual-NH

120 thermostat developed by Son et al. ${ }^{33}$ that improves equipartitioning of kinetic energy, which proved to

121 be useful particularly for simulations with CL\&P-D ${ }_{k}{ }^{25}$

122 For both the Coulombic and dispersion interactions, we set the cut-off distance to $12 \AA$. Long-range

123 Coulombic interactions were computed using the particle-particle particle-mesh (PPPM) solver.

124 The original CL\&P and CL\&P-D $k$ FF parameter values for the considered ions can be found in ref ${ }^{34}$ 125 and their original references in ref ${ }^{25}$. Atomic polarizability values, needed in the CL\&P-D model to $^{2}$

126 calculate the charges of the Drude particles, were adopted from Schröder et al. ${ }^{24}$ and corresponded to

127 averaged values for individual atom types present in ILs.

128 The atomic Lennard-Jones pair potential parameters present in the original fixed-charge CL\&P FF 129 already implicitly account to some extent for the polarizability effects. ${ }^{35}$ To avoid double-counting of 130 those when CL\&P is combined with the Drude model (CL\&P-D $k$ ), and to subtract the implicit

131 polarization component from the attractive part of LJ, Pádua and his colleagues ${ }^{20,27,35}$ proposed a 132 methodology that determines scaling factors $(k)$ for the energetic LJ parameters $\varepsilon_{\mathrm{LJ}}$ between different 133 molecular fragments of ILs. This $k$-approach was considered in this work (hence the subscript $k$ in $\left.134 \mathrm{CL} \& \mathrm{P}-\mathrm{D}_{k}\right)$ and the respective $k$ values used to scale down the original $\varepsilon_{\mathrm{LJ}}$ parameters were taken from 135 the literature. ${ }^{20,25}$ As a result, the atomic polarization was exclusively described by the Drude 136 oscillators within the CL\&P-D $\mathrm{D}_{k}$ model. The original LJ parameters $\sigma_{\mathrm{LJ}}$ from CL\&P were kept 137 unchanged..$^{25}$

\section{$138 \quad 2.2$ Evaluation of $\boldsymbol{T}_{\mathrm{g}}$}

139 Two approaches were considered in this work to obtain glass transition temperatures from MD 140 simulations: from the temperature-trend of (i) densities and (ii) diffusivities calculated using MD 141 within the same trajectory. Both approaches are described in more detail below. 
143 Density $(\rho)$ data simulated for each IL and each FF model in a temperature range of 100 to $400 \mathrm{~K}$ were 144 divided into two branches representing the glassy (approximately 100 to $200 \mathrm{~K}$ ) and liquid 145 (approximately 250 to $400 \mathrm{~K}$ ) phases. In some cases, the upper temperature bound of the glassy phase 146 and/or the lower bound of the liquid phase were modified in order to exclude outliers or data points 147 belonging to the vicinity of the $T_{\mathrm{g}}$ region, or to include more data points that still follow the linear 148 temperature-trend of the respective phase. A detailed overview of the data points used/excluded from 149 the linear regression and subsequent determination of $T_{\mathrm{g}}$ is shown in Figure $\mathrm{S} 1$. These branches of 150 simulated density points were linearly interpolated against the temperature. Subsequently, the 151 intersection of the obtained fitted lines determined the location of $T_{\mathrm{g}}$, as illustrated in Figure $\mathrm{S} 1$. The 152 uncertainty of the simulated density data due to inefficient MD sampling was quantified using the

153 block-averaging technique. ${ }^{36}$ However, the uncertainty of the linear regressions used to determine $T_{\mathrm{g}}$ 154 was found to be larger than the uncertainty estimated by the block-averaging technique. Therefore, the 155 final uncertainty in $T_{\mathrm{g}}{ }^{\rho}$ was calculated based on the error propagation law from the uncertainties of the 156 linear regressions. ${ }^{37}$

\subsection{2 $T_{\mathrm{g}}$ from diffusivities}

158 The self-diffusivities $(D)$ at each simulation temperature were determined from the simulated equilibrium trajectories via the recommended Einstein relation: ${ }^{38}$

$$
D=\frac{1}{6} \lim _{t \rightarrow \infty} \frac{\mathrm{d}}{\mathrm{d} t}\left\langle|\mathbf{r}(t)-\mathbf{r}(0)|^{2}\right\rangle
$$

161 using the time-dependent mean-square displacement (MSD), that is, $\operatorname{MSD}(t)=\left\langle|\mathbf{r}(t)-\mathbf{r}(0)|^{2}\right\rangle$,

162 calculated from the molecular positions (r). The employed procedure to calculate $T_{\mathrm{g}}$ from $D$ was similar 163 to that used in the case of densities: temperature dependent $\log (D)$ values, calculated from the slopes 
164 of MSD versus time, ${ }^{38}$ were divided into the glassy and liquid phases (roughly $100-200 \mathrm{~K}$ and 250

$165400 \mathrm{~K}$, respectively). $T_{\mathrm{g}}$ was again evaluated from the intersection of the respective linear regressions.

166 Again, the statistical uncertainty of the simulated data points turned out to be generally lower than that

167 of the linear fit, so the final uncertainty in $T_{\mathrm{g}}{ }^{D}$ was calculated from the latter. An overview of the data

168 points used/excluded from the linear regression and the determined $T_{\mathrm{g}}$ values are shown in Figure $\mathrm{S} 2$.

169 For each IL, the self-diffusivities and, hence, the corresponding $T_{\mathrm{g}}$ values were calculated separately

170 for its cations, anions, and the ion pairs as a whole. However, no notable differences were observed

171 among the $T_{\mathrm{g}}$ data determined from $D$ of these three entities, as can be seen by comparing Figures $\mathrm{S} 2$,

172 S3 and S4 in the Supporting Information. Therefore, we arbitrarily used those for cations, $D_{\text {cat, }}$ to

173 determine, assess, and discuss $T_{\mathrm{g}}{ }^{D}$ estimates based on diffusivities in this work. 


\section{RESULTS AND DISCUSSION}

176 Experimental glass transition temperatures and their counterparts calculated in this work $\left(T_{\mathrm{g}}{ }^{\rho}\right.$ and $\left.T_{\mathrm{g}}{ }^{D}\right)$

177 using the three different FF models for all five ILs are shown in Table 2 and displayed in Figure S5

178 and Figure S6, respectively, in the Supporting Information. Their determination from temperature-

179 dependent data is summarized in Figures S1 and S2 in the Supporting Information; a sample of the $T_{\mathrm{g}}$

180 determination for $[\mathrm{bmIm}]\left[\mathrm{NTf}_{2}\right]$ is also shown in Figure 5. The deviations of the calculated $T_{\mathrm{g}}{ }^{\rho}$ and $T_{\mathrm{g}}{ }^{D}$

181 data from the experimental ones are shown in Figure 1 and Figure 3 below, respectively.

\section{$182 \quad 3.1 \quad$ Glass transitions from densities}

183 As can be seen in Table 2 and Figure $1, T_{\mathrm{g}}{ }^{\rho}$ values from CL\&P are systematically overestimated by

184 tens of Kelvins (up to ca. $65 \mathrm{~K}$ ), with an absolute average deviation (AAD) of $37 \mathrm{~K}$. This is in 185 correspondence with our recent findings on $T_{\mathrm{g}}$ values for a series of [emIm]-based ILs. ${ }^{1}$ Moreover, the

$186 T_{\mathrm{g}}$ predictions from CL\&P show the largest scatter in absolute deviations (16 K; see Table 2).

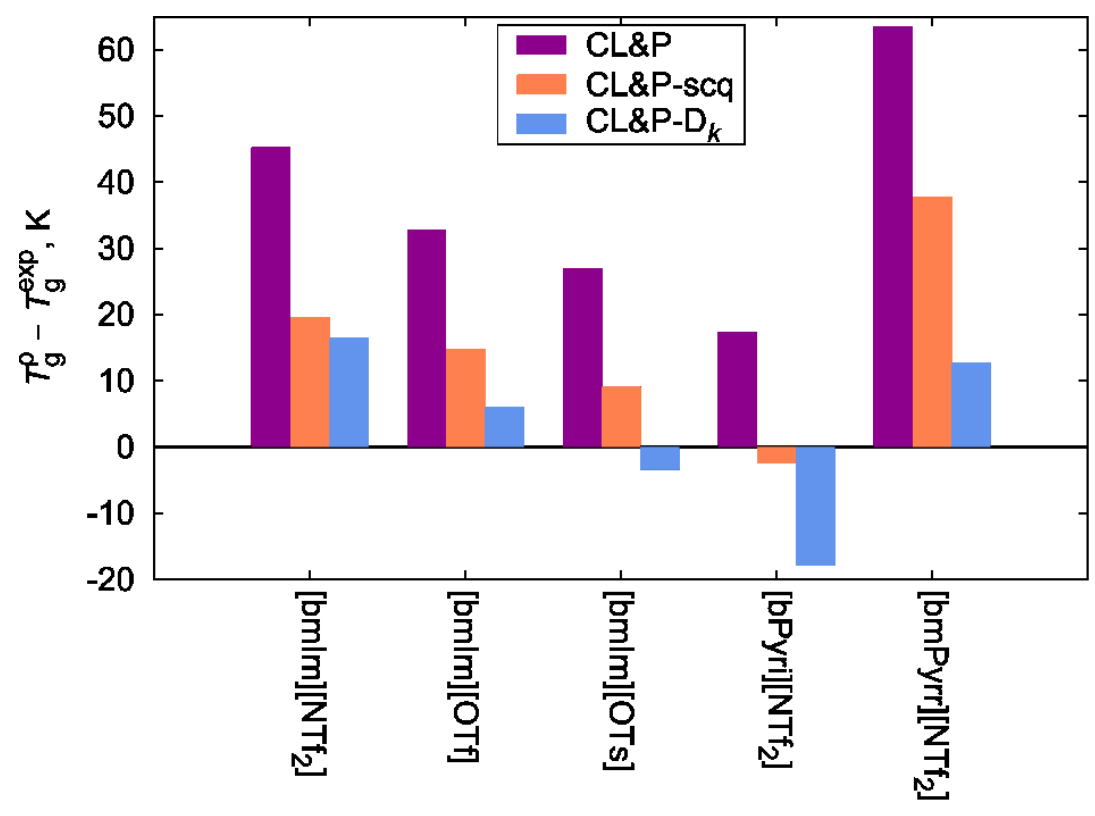

188 Figure 1. Deviations of $T_{\mathrm{g}}{ }^{\rho}$ (calculated by MD from temperature-trend in densities) from experimental 189 data for all 5 ILs. 
190 CL\&P-scq also tended to overestimate $T_{\mathrm{g}}$ values (for 4 out of 5 ILs), but the corresponding AAD was

191 significantly lower $(17 \mathrm{~K})$, revealing a better quantitative performance of this scaled yet still non192 polarizable variant of CL\&P. Particularly notable is the quantitative performance of CL\&P-scq for $193\left[\right.$ bPyri] $\left[\mathrm{NTf}_{2}\right]($ deviation around $-2 \mathrm{~K})$.

194 Unlike the two non-polarizable models, CL\&P-D $k$ did not show a clear tendency to overpredict $T_{\mathrm{g}}$, as 195 an underestimation was found for 2 out of $5 \mathrm{ILs}$ : [bmIm][OTs] and [bPyri][NTf 2 ]. AAD for CL\&P-D $k$ 196 was $11 \pm 6 \mathrm{~K}$, making this $\mathrm{FF}$ the most accurate, robust and the least scattered approach for $T_{\mathrm{g}}{ }^{\rho}$ 197 predictions of ILs among the FFs tested in this work. Nearly quantitative predictions were obtained for $198[\mathrm{bmIm}][\mathrm{OTf}]$ and $[\mathrm{bmIm}][\mathrm{OTs}]$, as can be seen in Figure 1.

$199 T_{\mathrm{g}}{ }^{\rho}$ estimates from CL\&P-scq compete with those from CL\&P-D $\mathrm{D}_{k}$ in terms of quantitative accuracy 200 (AADs $17 \mathrm{~K}$ and $11 \mathrm{~K}$, respectively); moreover, CLP-scq simulations were significantly less 201 computationally expensive (roughly 4 to 5-fold).

202 For each IL, $T_{\mathrm{g}}{ }^{\rho}$ estimate from CL\&P is always the highest one, followed by that from CL\&P-scq, 203 whereas $T_{\mathrm{g}}{ }^{\rho}$ from CL\&P-D $k$ is always the lowest (that is, $T_{\mathrm{g}}{ }^{\mathrm{CL} \& \mathrm{P}}>T_{\mathrm{g}}{ }^{\mathrm{CL} \& \mathrm{P}-\mathrm{scq}}>T_{\mathrm{g}}{ }^{\mathrm{CL} \& \mathrm{P}-\mathrm{D} k}$ ). Moreover, 204 the results of this work allow for evaluating the following average scaling factors between $T_{\mathrm{g}}$ values 205 predicted by the different FF models:

$$
\begin{aligned}
& T_{\mathrm{g}}^{\text {CL\&P-scq }}=(0.91 \pm 0.01) T_{\mathrm{g}}{ }^{\text {CL\&P }} ; \\
& T_{\mathrm{g}}^{\text {CL\&P-D } k}=(0.85 \pm 0.03) T_{\mathrm{g}}^{\text {CL\&P }} \\
& T_{\mathrm{g}}^{\text {CL\&P-D } k}=(0.94 \pm 0.03) T_{\mathrm{g}}^{\text {CL\&P-scq }} .
\end{aligned}
$$

209 Therefore, for each individual IL, magnitude of the deviation between the predicted and experimental $210 T_{\mathrm{g}}{ }^{\rho}$ data is largely determined by the exact location of the latter. For example, if CL\&P largely 211 overestimates $T_{\mathrm{g}}$ for an IL (as in the case of $[\mathrm{bmIm}]\left[\mathrm{NTf}_{2}\right]$ ), CL\&P-scq and CL\&P-D $k$ would probably 212 decrease this error. On the other hand, if CL\&P shows a relatively low error for an IL (as for 
213 [bPyri][ $\left.\mathrm{NTf}_{2}\right]$ ), a decrease in predicted $T_{\mathrm{g}}$ achieved by CL\&P-scq and CL\&P-D $\mathrm{D}_{k}$ may lead to a larger

214 (negative) error compared to CL\&P, as really seen for CL\&P- $\mathrm{D}_{k}$ and [bPyri][NTf 2 .

215 Fairly constant ratios between $T_{\mathrm{g}}$ values calculated with the different FF models are further reflected

216 in very similar slopes of the linear correlation between $T_{\mathrm{g}}{ }^{\rho}$ and $T_{\mathrm{g}}{ }^{\text {exp }}$, as illustrated in Figure 2, although

217 the $T_{\mathrm{g}}$ predictions from the different FFs generally show different levels of correlation with their

218 experimental counterparts, as measured by the coefficient of determination $\left(R^{2}\right.$ in Figure 2$)$. Based on

219 the obtained $R^{2}$ values, CL\&P-D $k$ shows the best capability to rank the ILs according to their $T_{\mathrm{g}}$

$220\left(R^{2}=0.40\right)$ among the tested FFs, followed by CL\&P-scq and CL\&P $\left(R^{2}=0.35\right.$ and 0.18 , respectively $)$.

221 However, such $R^{2}$ values indicate a relatively weak correlation between the calculated and

222 experimental $T_{\mathrm{g}}$ data.

223 The IL $[\mathrm{bmIm}][\mathrm{OTs}]$ shows the largest experimental $T_{\mathrm{g}}$ from the test set. All the three FF models

224 qualitatively correctly predicted this fact. However, CL\&P and CL\&P-scq did not qualitatively capture

225 the fact that [bmPyrr][NTf2] shows one of the lowest experimental $T_{\mathrm{g}}$; they surprisingly predicted that

226 this IL has one of the largest $T_{\mathrm{g}}$.

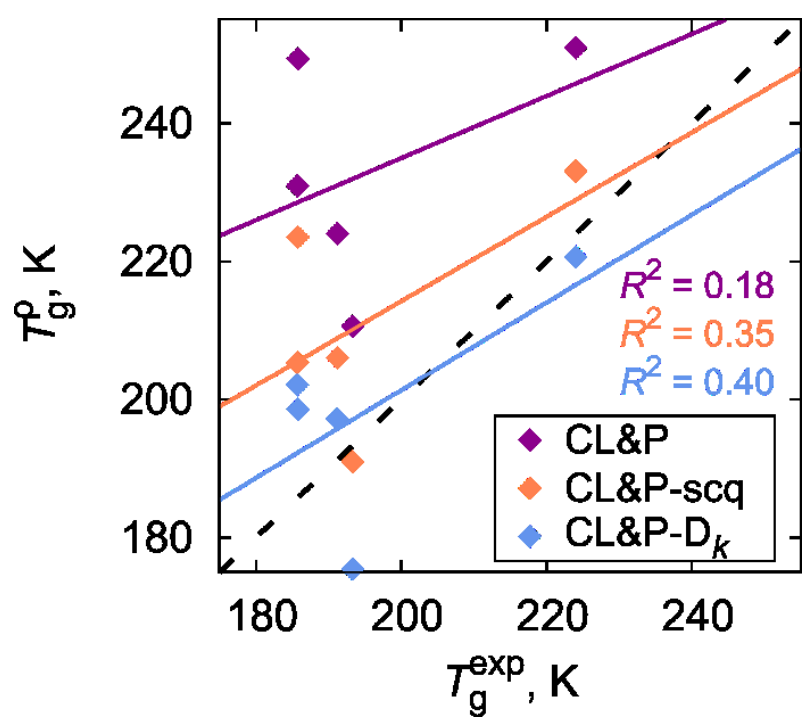

228 Figure 2. Diagonal comparison of experimental $T_{\mathrm{g}}$ and $T_{\mathrm{g}}{ }^{\rho}$ calculated from temperature-trend in 229 densities. 
TABLE 2

232 Comparison of Experimental Glass Transition Temperatures $T_{\mathrm{g}}$ (in $\mathrm{K}$ ) with Their Counterparts 233 Obtained from MD Simulations with Either Non-Polarizable (CL\&P and CL\&P-scq) or Polarizable $234($ CL\&P-D $k)$ Force Field Models.

\begin{tabular}{|c|c|c|c|c|c|c|c|}
\hline \multirow{2}{*}{ Ionic liquid } & \multirow{2}{*}{$T_{\mathrm{g}}^{\exp }$} & \multicolumn{3}{|c|}{$T_{\mathrm{g}}^{\mathrm{MD}}$ from $\rho$} & \multicolumn{3}{|c|}{$T_{\mathrm{g}}^{\mathrm{MD}}$ from $D_{\text {cat }}$} \\
\hline & & CL\&P & CL\&P-scq & CL\&P-D $k$ & CL\&P & CL\&P-scq & CL\&P-D $k$ \\
\hline$[\mathrm{bmIm}]\left[\mathrm{NTf}_{2}\right]$ & $185.7^{\mathrm{a}}$ & $231 \pm 7$ & $205 \pm 7$ & $202 \pm 12$ & $285 \pm 17$ & $255 \pm 23$ & $227 \pm 22$ \\
\hline [bmIm][OTf] & $191.2^{\mathrm{b}}$ & $224 \pm 13$ & $206 \pm 14$ & $197 \pm 17$ & $296 \pm 34$ & $262 \pm 23$ & $236 \pm 17$ \\
\hline$[\mathrm{bmIm}][\mathrm{OTs}]$ & $224.0^{\mathrm{c}}$ & $251 \pm 17$ & $233 \pm 8$ & $221 \pm 9$ & $260 \pm 24$ & $247 \pm 24$ & $235 \pm 27$ \\
\hline$[$ bPyri $]\left[\mathrm{NTf}_{2}\right]$ & $193.3^{\mathrm{d}}$ & $211 \pm 9$ & $191 \pm 7$ & $175 \pm 11$ & $228 \pm 22$ & $215 \pm 21$ & $184 \pm 20$ \\
\hline$[\mathrm{bmPyrr}]\left[\mathrm{NTf}_{2}\right]$ & $185.8^{\mathrm{a}}$ & $249 \pm 16$ & $224 \pm 7$ & $199 \pm 15$ & $250 \pm 22$ & $240 \pm 16$ & $237 \pm 32$ \\
\hline $\operatorname{AAD}(K)^{\mathrm{e}}$ & & $37 \pm 16$ & $17 \pm 12$ & $11 \pm 6$ & $68 \pm 30$ & $48 \pm 22$ & $31 \pm 18$ \\
\hline
\end{tabular}

${ }^{a}$ Averaged over the multiple experimental data available in the ILThermo database ${ }^{39}$ (excluding obvious outliers).

$236{ }^{\mathrm{b}} \operatorname{Ref}^{40}$

$237{ }^{\mathrm{c}} \operatorname{Ref}^{41}$

$238{ }^{\mathrm{d}} \operatorname{Ref}^{42}$

$239{ }^{\mathrm{e}} \mathrm{AAD}$ is the average absolute deviation: $\mathrm{AAD}=N^{-1} \sum_{i=1}^{N}\left|T_{\mathrm{g}, i}^{\mathrm{MD}}-T_{\mathrm{g}, i}^{\mathrm{exp}}\right|$, where $N$ denotes the number of ILs. The number 240 after " \pm “" represents standard deviation of the individual absolute deviations.

\section{$242 \quad 3.2 \quad$ Analysis of the simulated densities}

243 It can be seen in Figure S1 that the CL\&P-scq model generally decreases the density of the glass and,

244 in particular, the liquid phase compared to the original CL\&P model, which seems intuitive as the 245 charge-downscaling weakens the Coulombic forces between the cations and anions and under-binds 246 the bulk phase. Regarding CL\&P-D $k$, although it also decreases densities of the liquid, densities on the 247 glass branch are higher compared to those of both CL\&P and CL\&P-scq. This means that the glassy 248 state simulated by CL\&P-D $\mathrm{D}_{k}$ is denser, indicating stronger interactions predicted by CL\&P-D $\mathrm{D}_{k}$ in this 249 temperature region. This behavior is not seen for CL\&P-scq, and a possible explanation could be that 
the simple charge downscaling done in CL\&P-scq is not compensated by any other effect, whereas in

251 CL\&P-D $k$ the charge subtracted from an atom is not lost but assigned to the corresponding Drude 252 particle.

253 Indeed, we demonstrate in Figure S7 that the electrostatic interactions among the permanent atomic 254 charges are the most important contribution to the cohesion of bulk glassy phases at $150 \mathrm{~K}$ when the 255 CL\&P-D $\mathrm{D}_{k}$ model is applied. Absolute cohesion due to the electrostatics rises by $14 \%$ on average upon 256 the transition from CL\&P-D to CL\&P-D $k$. On the other hand, the dispersion contribution to the overall 257 cohesion wanes appreciably (by 22\%) when the CL\&P-D model is invoked. This seems 258 counterintuitive, given that the $\mathrm{CL} \& \mathrm{P}-\mathrm{D}_{k}$ glasses are generally denser than those of CL\&P. However, 259 such a drop of the dispersion interactions in the polarizable glasses can be traced to downscaling the $260 \varepsilon \mathrm{EJ}$ parameter of the LJ potential (see Section 2.1), leading to a decrease of magnitude of all dispersion 261 interactions and/or to lowering the repulsion branch of the LJ potential curve. For the latter, stronger 262 electrostatic interactions are, in some cases, capable of pushing non-bonded atoms, possessing the most 263 significant partial charges, closer to one another, invoking even a weakly repulsing dispersion regime 264 for the closest atomic contacts (but still with a negative interaction energy). This is demonstrated in 265 Figure S8 in the Supporting Information using radial distribution functions (RDFs), $g(r)$, of C-O pair 266 interactions for $[\mathrm{bmIm}]\left[\mathrm{NTf}_{2}\right]$ and $[\mathrm{bmPyrr}]\left[\mathrm{NTf}_{2}\right](\mathrm{C}$ represents selected carbon atoms of the cation 267 ring, whereas $\mathrm{O}$ denotes the oxygen atoms of [ $\left.\mathrm{NTf}_{2}\right]$; see Figure $\mathrm{S} 8$ for details). It can be seen that the 268 peak maximum (3.19 $\AA$ ) obtained from $C L \& \mathrm{P}-\mathrm{D}_{k}$ at $150 \mathrm{~K}$ for the first coordination shell of $269[\mathrm{bmIm}]\left[\mathrm{NTf}_{2}\right]$ is located at a mutual distance lower than the cross LJ parameter $\sigma_{\mathrm{LJ}}(\mathrm{C}, \mathrm{O})=3.25 \AA$, 270 whereas that from CL\&P (3.28 $\AA)$ is slightly higher than $\sigma_{\mathrm{LJ}}(\mathrm{C}, \mathrm{O})$. This effect holds qualitatively even 271 at an elevated temperature of $360 \mathrm{~K}$. In the case of [bmPyrr][NTf 2 , the $\mathrm{C}-\mathrm{O}$ peak position $(3.37 \AA)$

272 from $C L \& P-D_{k}$ at $150 \mathrm{~K}$ is slightly higher than the corresponding $\sigma_{\mathrm{LJ}}(\mathrm{C}, \mathrm{O})$ value of $3.33 \AA$, but is still 273 much closer to $\sigma_{\mathrm{LJ}}(\mathrm{C}, \mathrm{O})$ than that from CL\&P $(3.54 \AA)$, again indicating a more intensive packing of 274 the CL\&P-D $k$ glass. Since the closest contact distances in bulk glassy [bmPyrr][NTf $\left.\mathrm{N}_{2}\right]$ simulated with 
275 CL\&P-D ${ }_{k}$ still range in the attractive dispersion regime, shrinking of the glass due to turning-on the

276 CL\&P- $\mathrm{D}_{k}$ model can be there more pronounced, unlike the case of $[\mathrm{bmIm}]\left[\mathrm{NTf}_{2}\right]$ where the already

277 repulsive dispersion hinders further compression of the material.

278 Unfortunately, there are no experimental data for the sub-cooled liquid and glassy phases of the

279 considered ILs in literature. To gain an insight about the possible accuracy of our simulations of bulk

280 densities at low temperatures, we culled the experimental density data from the ILThermo database, ${ }^{39}$

281 and extrapolated those high-temperature data down to $200 \mathrm{~K}$ (only to $220 \mathrm{~K}$ for [bmIm][OTs]) using

282 the isobaric coefficient of thermal expansion, $\alpha_{p}$, defined as:

$$
\alpha_{p}=-\frac{1}{\rho}\left(\frac{\partial \rho}{\partial T}\right)_{p} .
$$

284 Note that for deriving the reference data, we accepted only those entries with reported uncertainties

285 lower than $1 \mathrm{~kg} \mathrm{~m}^{-3}$ for $[\mathrm{bmIm}]\left[\mathrm{NTf}_{2}\right],[\mathrm{bmIm}][\mathrm{OTf}]$, and [bmPyrr][NTf 2 . Due to limited data

286 availability, we loosened this threshold to $2 \mathrm{~kg} \mathrm{~m}^{-3}$ for $[\mathrm{bmIm}][\mathrm{OTs}]$, and [bPyri] $\left[\mathrm{NTf}_{2}\right]$. The

287 extrapolated experimental data could be considered as fairly reliable above $200 \mathrm{~K}$ ( $230 \mathrm{~K}$ for $288[\mathrm{bmIm}][\mathrm{OTs}])$, being above the experimental $T_{\mathrm{g}}$. All such selected experimental density data, along 289 with the references to the original publications are summarized in Figures S9-S13.

290 Comparison of the calculated an experimental densities in Figures S9-S13 reveals that both CL\&P and

291 CL\&P-D $\mathrm{D}_{k}$ models underestimate the bulk densities consistently at all temperatures for $[\mathrm{bmIm}][\mathrm{OTf}]$,

$292[\mathrm{bmIm}][\mathrm{OTs}]$, and $[\mathrm{bPyri}]\left[\mathrm{NTf}_{2}\right]$. Both predicted density data sets are systematically overestimated for

$293[\mathrm{bmIm}]\left[\mathrm{NTf}_{2}\right]$, whereas the sign of the difference between theory and experiment depends on

294 temperature and computational model for [bmPyrr][ $\left.\mathrm{NTf}_{2}\right]$. In all cases except [bmPyrr][NTf 2$], \mathrm{CL} \& \mathrm{P}$

295 yields higher densities than the CL\&P-D $\mathrm{D}_{k}$ model. The comparison of CL\&P densities with extrapolated

296 experimental data for liquid becomes misleading below $230 \mathrm{~K}$ as the simulations already predict the

297 trend shift due to vitrification in that temperature region. In the low-temperature region $(230-270 \mathrm{~K})$, 
AARD of the calculated densities amounts to $2.7 \%$ and $3.2 \%$ for CL\&P model and CL\&P-D $\mathrm{D}_{k}$ models, respectively, whereas these AARD shift to $2.5 \%$ and $3.7 \%$ above $270 \mathrm{~K}$ on average.

300

\subsection{Glass transitions from diffusivities}

302 For $T_{\mathrm{g}}$ calculated from (cation) diffusivities, qualitative trends similar to those for $T_{\mathrm{g}}{ }^{\rho}$ are seen. Namely, 303 the following sequence can again be seen for the $T_{\mathrm{g}}$ results calculated from the different FFs for each

304 IL: $T_{\mathrm{g}}{ }^{\text {CL\&P }}>T_{\mathrm{g}}{ }^{\text {CL\&P-scq }}>T_{\mathrm{g}}$ CL\&P-D $k$. However, the average scaling factors between $T_{\mathrm{g}}$ data predicted by 305 the different FF models are somewhat more scattered and differ more for individual ILs compared the 306 obtained density-based $T_{\mathrm{g}}$ discussed in the previous section:

$$
T_{\mathrm{g}}{ }^{\text {CL\&P-scq }}=(0.93 \pm 0.03) T_{\mathrm{g}}{ }^{\text {CL\&P }}
$$$$
T_{\mathrm{g}}^{\mathrm{CL \& P}-\mathrm{D} k,}=(0.85 \pm 0.06) T_{\mathrm{g}}^{\mathrm{CL \& P}}
$$$$
T_{\mathrm{g}}^{\text {CL\&P-D } k}=(0.92 \pm 0.05) T_{\mathrm{g}}{ }^{\text {CL\&P-scq }} .
$$

310 Unlike for $T_{\mathrm{g}}{ }^{\rho}$, no correlation between experimental $T_{\mathrm{g}}$ and $T_{\mathrm{g}}{ }^{D}$ is found, as shown in Figure 4 and

311 indicated by the effectively zero $R^{2}$ values for all three FFs therein. This means that $T_{\mathrm{g}}$ estimates based

312 on simulated diffusivities are inapplicable for ranking ILs even with CL\&P-D $\mathrm{D}_{k}$, at least within the

313 considered test set of ILs.

314 In terms of quantitative aspects, it can be seen in Table 2 and Figure 3 that $T_{\mathrm{g}}{ }^{D}$ estimates generally

315 show larger deviations from experimental data than those based on densities. AADs for CL\&P, CL\&P-

316 scq and CL\&P-D $\mathrm{D}_{k}$ now reach 68,48 and $31 \mathrm{~K}$, respectively, whereas it is only 37,17 and $11 \mathrm{~K}$ for $T_{\mathrm{g}}{ }^{\rho}$.

317 Note that the ILs $[\mathrm{bmIm}]\left[\mathrm{NTf}_{2}\right]$ and $[\mathrm{bmIm}][\mathrm{OTf}]$ are the main contributors to these large AADs; for

318 these ILs, CL\&P overestimates $T_{\mathrm{g}}$ by approximately $100 \mathrm{~K}$. The best predictions are again achieved 319 from CL\&P-D $k$, followed by CL\&P-scq and CL\&P. 


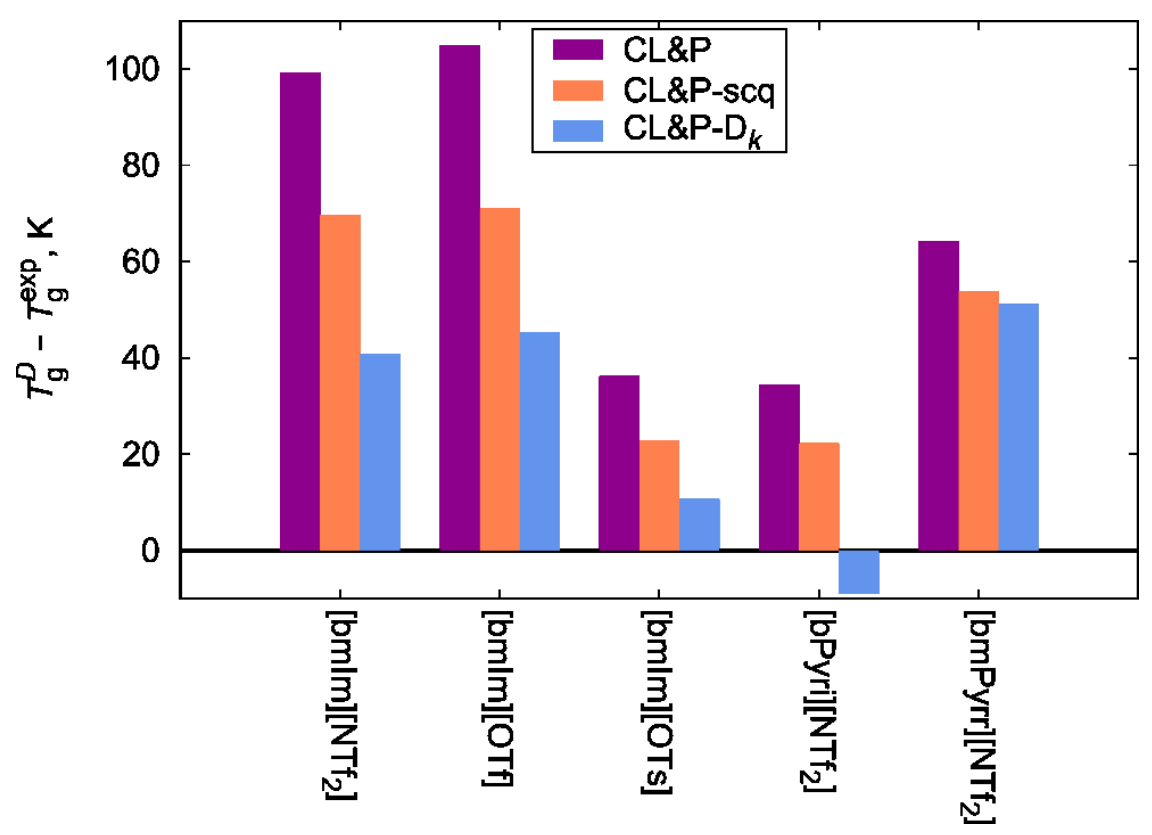

321 Figure 3. Deviations of $T_{\mathrm{g}}{ }^{D}$ (calculated by MD from temperature-trend in cation diffusivities) from 322 experimental data for all 5 ILs.

323 The uncertainties of the individual $T_{\mathrm{g}}{ }^{D}$ values reported in Table 2 are also larger than their $T_{\mathrm{g}}{ }^{\rho}$

324 counterparts. This is associated by the larger scatter of the simulated temperature-dependent diffusivity

325 data compared to those on densities (compare Figures S1 and S2 in the Supporting Information), which

326 can be attributed to the fact that the calculated diffusivities are not equilibrium averages over the

327 simulated MD trajectory and are thus more challenging to compute accurately, especially in the context

328 of obtaining a smooth temperature dependence. ${ }^{38}$ On the other hand, the simulated diffusivities show

329 a more significant temperature-trend shift upon melting of the glassy state in most cases compared to

330 densities, as can be seen in Figures S1 and S2. 


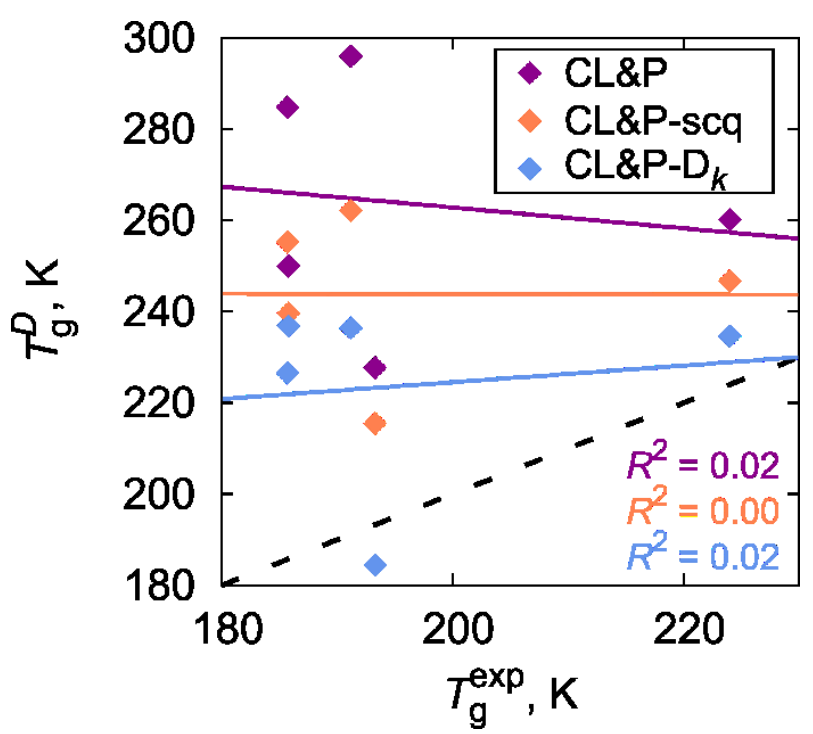

332 Figure 4. Diagonal comparison of experimental $T_{\mathrm{g}}$ and $T_{\mathrm{g}}{ }^{D}$ calculated from temperature-trend in cation 333 diffusivities.

\subsection{Self-diffusivities of the sub-cooled liquid}

336 Interestingly, temperature trends of the diffusivities computed from the non-polarizable and polarizable

337 simulations differ qualitatively. A closer inspection of Figure S2 reveals that both the CL\&P-D $k$ and

338 CL\&P-scq models yield higher $D$ values for the liquid at elevated temperatures, which justifies the 339 motivation for introducing the polarizable simulations for ILs. ${ }^{20}$ Upon cooling the liquid within CL\&P-

$340 \mathrm{D}_{k}$, however, there is another well-observable trend shift of $D$ in the region $250-350 \mathrm{~K}$ for all the

341 considered liquids, attenuating the ionic self-diffusivity faster in CL\&P-D than it happens in CL\&P.

342 As a result, the self-diffusivities simulated for glasses using CL\&P-D $\mathrm{D}_{k}$ are often lower than those

343 resulting from the original CL\&P model. In our test set, we observed this behavior for 3 out of 5

344 species, namely for $[\mathrm{bmIm}]\left[\mathrm{NTf}_{2}\right],[\mathrm{bmIm}][\mathrm{OTf}]$, and [bPyri] $\left[\mathrm{NTf}_{2}\right]$.

345 This behavior of the simulated amorphous solids does not always correlate with the trends of predicted

346 densities (a density increase of the glass in CL\&P-D $k$ does not necessarily lead to a drop of its self-

347 diffusivity). To investigate this phenomenon further and to interpret it via any suitable structural

348 aspects, we performed simulations of small-angle neutron diffraction for liquids and glasses at various 
temperatures. Static structure factors for $[\mathrm{bmIm}]\left[\mathrm{NTf}_{2}\right]$ were calculated on the simulated trajectories

350 (10 ns period for CL\&P and $5 \mathrm{~ns}$ for CL\&P-D $k$ ) by the Diffraction utility, ${ }^{43}$ using coherent scattering

351 lengths for the elements from NIST, ${ }^{44}$ the computational setup of which was previously validated for 352 ILs. ${ }^{45}$

353 Figure 5 compares the structure factors $(S)$ as functions of the reciprocal length $(q)$ at various 354 temperatures. All signals exhibit a broad peak around $q=1.3-1.4 \AA^{-1}$, moving to smaller reciprocal 355 lengths upon heating. This can be interpreted as an imprint of the closest atomic contacts (size of the 356 first coordination shell is then $2 \pi / 1.4 \AA^{-1}=4.5 \AA$ ) in the bulk amorphous phases. Further, there is an 357 undeniable shoulder peak below $0.9 \AA^{-1}$ for the non-polarizable simulations of liquid, which can be 358 attributed to a well-preserved cage structure of the second coordination shells (characteristic size is $3592 \pi / 0.9 \AA^{-1}=7.0 \AA$ ) in ILs even at $400 \mathrm{~K}$. This is usually interpreted as the principal artifact of non360 polarizable simulations of ILs causing the massive underestimation of the predicted self-diffusivities 361 of ions. ${ }^{10,12,20}$ Note that there are no such analogous peaks for the liquid above $280 \mathrm{~K}$ in the CL\&P-D $k$ 362 results.

363 In contrast, significant features of $S(q)$ appear in the temperature range 220-280 K (representing in fact 364 sub-cooled liquid $\left.[\mathrm{bmIm}]\left[\mathrm{NTf}_{2}\right]\right)$, for CL\&P-D $\mathrm{D}_{k}$ trajectories. A sharp peak is manifested around $0.4 \AA^{-1}$ 365 at $250 \mathrm{~K}$, becoming broader as the temperature increases. Furthermore, there is another well366 pronounced peak above $0.2 \AA^{-1}$, being the most intense at $260 \mathrm{~K}$. Such features of the structure factor 367 indicate some form of longer-range ordering with characteristic size of 16 and $31 \AA$, respectively.

368 Note that these alterations of the structure factor appear in the exactly same temperature region, where 369 the ionic self-diffusivities exhibit the steepest increase with temperature. A possible explanation could 370 thus be that after melting of the glass using CL\&P-D $\mathrm{D}_{k}$, the polarizable liquid phase organizes itself into 371 distinct domains (spanning more than only two coordination shells), which restrain the ionic mobility 372 at first, but the reorganization of which results in a steep increase of the mobility upon heating. Above $373280 \mathrm{~K}$, existence of such domains becomes improbable according to the CL\&P-D $k$ simulations, leading 
374 to a shift of the diffusivity vs. temperature trend, and also to self-diffusivities already larger than in the

375 non-polarizable model.

376 Trying to clarify the origin of these structural features, we calculated a series of RDFs for various

377 different pairs of atoms within $[\mathrm{bmIm}]\left[\mathrm{NTf}_{2}\right]$ with CL\&P-D $k$ using an increased cut-off value of $30 \AA$

378 to cover the long-range structural features of interest. Two different temperatures were considered:

$379250 \mathrm{~K}$ corresponding the mentioned sharp peak around $0.4 \AA^{-1}$, and an elevated temperature of $360 \mathrm{~K}$,

380 at which such structural features should be eliminated. However, the analysis shows no notable

381 qualitative or quantitative differences between the RDFs calculated at both temperatures.

382
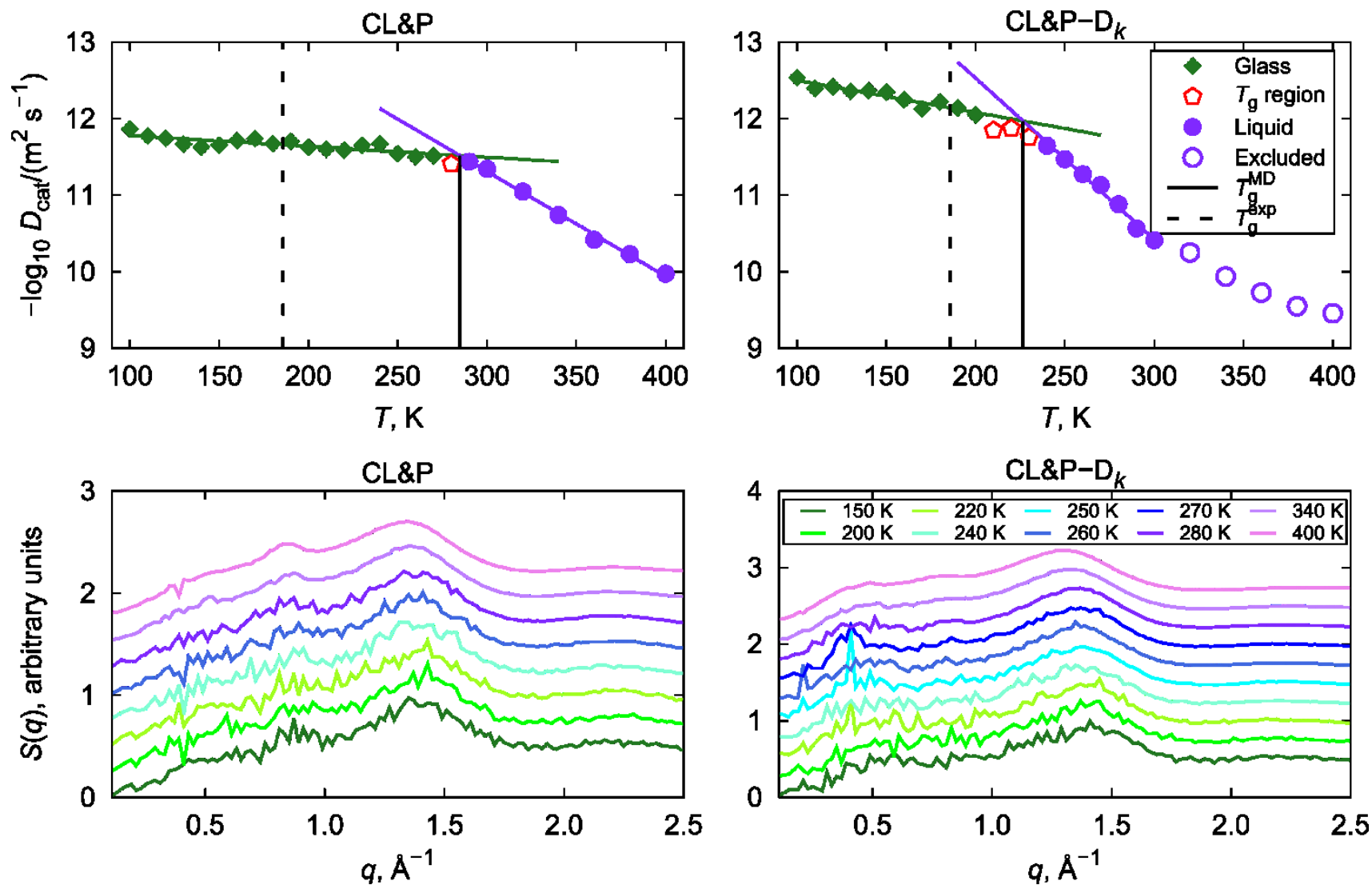

384 Figure 5. Determination of glass transition temperatures from simulated cation diffusivities (top) and 385 structure factors, $S(q)$, calculated to mimic the small-angle neutron diffraction (bottom) considering 386 the MD trajectories simulated for $[\mathrm{bmIm}]\left[\mathrm{NTf}_{2}\right]$ with the CL\&P (left) and CL\&P-D $k$ (right) force 387 fields. Note that the individual $S(q)$ curves are shifted artificially with respect to the corresponding 388 temperature for a better readability of the plots. 


\section{CONCLUSIONS}

390 The glass transition temperatures of five ILs obtained from MD simulations using three different FF

391 models were evaluated and compared with the experimental counterparts. In all cases, the $T_{\mathrm{g}}$ values

392 were determined using the temperature-trend shift method from both densities and cation diffusivities

393 separately.

394 The CL\&P-D $k$ FF model shows the best general performance in predicting $T_{\mathrm{g}}$, followed by the less 395 sophisticated non-polarizable scaled-charge CL\&P-scq and the original CL\&P models. Determination 396 of the glass transition temperature from the trend shift of the simulated densities is superior in terms of 397 accuracy to that based on the diffusivities, although the densities typically show a less abrupt trend 398 shift upon melting of the glassy state than the self-diffusivities do. It is the lesser scatter of the simulated 399 temperature-dependent densities (compared to the diffusivities) that renders the density analysis more 400 suitable for this purpose. The corresponding AAD values of the density-based predicted glass transition 401 temperatures are 11,17 and $37 \mathrm{~K}$ for CL\&P-D $k$, CL\&P-scq and CL\&P models, respectively. Such 402 deviations still exceed the typical experimental uncertainties, which range within a few units of Kelvin. 403 As a result, there is still space for a future search for a quantity that would exhibit an abrupt trend shift 404 upon the glassy transition and that would be extractable from polarizable molecular simulations with a 405 very low computational uncertainty to improve such predictions of the glass transition temperatures.

406 We also provide a few notes on some interesting observations regarding the simulated densities, 407 diffusivities and their temperature trends, attempting to explain and interpret them at the molecular 408 level. An indirect result of introducing the explicit polarizability (and scaling down the original 409 dispersion interactions) is an appreciable increase of the densities of the sub-cooled liquids and glasses

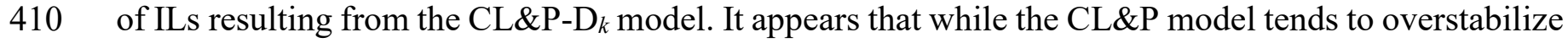
411 the first and second solvation shells of the ions in the liquid, even at elevated temperatures, the CL\&P$412 \mathrm{D}_{k}$ model predicts a longer-range ionic organization in the sub-cooled liquid, but just near-above the 
413 glass transition temperature. Existence of these domains can significantly lower the ionic self-

414 diffusivities for the highly sub-cooled liquids, as predicted with the CL\&P-D model.

415

416 ASSOCIATED CONTENT

\section{Supporting Information}

418 The supporting information is available free of charge at DOI:

419 1. Illustration of the trend-shift method for analyzing the simulated densities and diffusivities.

4202 2. Comparison of experimental and calculated glass transition temperatures by means of bar graphs.

421 3. Decomposition of the vaporization energy into individual mechanistic contributions.

422 4. Radial distribution functions of $\mathrm{C}-\mathrm{O}$ for $[\mathrm{bmIm}]\left[\mathrm{NTf}_{2}\right]$ and $[\mathrm{bmPyrr}]\left[\mathrm{NTf}_{2}\right]$.

423 5. Comparison of experimental and calculated densities of liquids.

AUTHOR INFORMATION

426 Corresponding Author

427 *E-mail: cervinkc@vscht.cz

428

429

\section{ACKNOWLEDGMENT}

430 The authors acknowledge financial support from the Czech Science Foundation (GACR No. 19431 04150Y). This work was supported by the Ministry of Education, Youth and Sports of the Czech 432 Republic through the e-INFRA CZ (ID:90140). 
436 1. Červinka, C.; Štejfa, V., Computational Assessment of the Crystallization Tendency of 1-Ethyl3-methylimidazolium Ionic Liquids. Physical Chemistry Chemical Physics 2021, 23, 4951-4962.

2. Zhang, Y.; Maginn, E. J., A comparison of methods for melting point calculation using molecular dynamics simulations. J. Chem. Phys. 2012, 136 (14), 144116.

$440 \quad 3 . \quad$ Habasaki, J.; Casalini, R.; Ngai, K. L., Molecular Dynamics Study of Thermodynamic Scaling of the Glass-Transition Dynamics in Ionic Liquids over Wide Temperature and Pressure Ranges. $J$. Phys. Chem. B 2010, 114 (11), 3902-3911. Transition in Ionic Liquids. Analyt. Sci. 2008, 24 (10), 1321-1327. 5. Zheng, L.; Luo, S.-N.; Thompson, D. L., Molecular dynamics simulations of melting and the glass transition of nitromethane. J. Chem. Phys. 2006, 124 (15), 154504.

6. Ghosh Dastidar, D.; Chakrabarti, G., Thermoresponsive Drug Delivery Systems, Characterization and Application. In Applications of Targeted Nano Drugs and Delivery Systems, Elsevier: 2019; pp 133-155. Dynamics Simulations. Macromolecules 1994, 27 (26), 7781-7784.

8. Rodríguez-Rivas, A.; Romero-Enrique, J. M.; Rull, L. F., Molecular simulation study of the glass transition in a soft primitive model for ionic liquids. Mol. Phys. 2019, 1-16.

9. Červinka, C.; Fulem, M., Structure and Glass Transition Temperature of Amorphous Dispersions of Model Pharmaceuticals with Nucleobases from Molecular Dynamics. Pharmaceutics 2021, $13(8)$.

10. Cadena, C.; Zhao, Q.; Snurr, R. Q.; Maginn, E. J., Molecular modeling and experimental studies of the thermodynamic and transport properties of pyridinium-based ionic liquids. J. Phys. Chem. B 2006, 110 (6), 2821-2832.

460 11. Kirchner, B.; Holloczki, O.; Lopes, J. N. C.; Padua, A. A. H., Multiresolution calculation of ionic liquids. Wiley Interdiscip. Rev. Comput. Mol. Sci. 2015, 5 (2), 202-214.

462 12. Maginn, E. J., Atomistic simulation of the thermodynamic and transport properties of ionic 463 liquids. Acc. Chem. Res. 2007, 40 (11), 1200-1207.

464 13. Canongia Lopes, J. N. A.; Padua, A. A. H., Nanostructural organization in ionic liquids. J. Phys. 465 Chem. B 2006, 110 (7), 3330-3335.

466 14. Köddermann, T.; Reith, D.; Ludwig, R., Comparison of Force Fields on the Basis of Various 467 Model Approaches-How To Design the Best Model for the [CnMIM][NTf2] Family of Ionic Liquids. 468 ChemPhysChem 2013, 14 (14), 3368-3374.

469 15. Son, C. Y.; McDaniel, J. G.; Schmidt, J. R.; Cui, Q.; Yethiraj, A., First-Principles United Atom 470 Force Field for the Ionic Liquid BMIM+BF4-: An Alternative to Charge Scaling. J Phys Chem B 2016, $471 \quad 120(14), 3560-3568$.

472 16. Zhang, Y.; Maginn, E. J., A Simple AIMD Approach to Derive Atomic Charges for Condensed Phase Simulation of Ionic Liquids. J. Phys. Chem. B 2012, 116 (33), 10036-10048.

17. Chaban, V., Polarizability versus mobility: atomistic force field for ionic liquids. Physical Chemistry Chemical Physics 2011, 13 (35), 16055-16062.

18. Vučemilović-Alagić, N.; Banhatti, R. D.; Stepić, R.; Wick, C. R.; Berger, D.; Gaimann, M. U.; Baer, A.; Harting, J.; Smith, D. M.; Smith, A.-S., Insights from molecular dynamics simulations on structural organization and diffusive dynamics of an ionic liquid at solid and vacuum interfaces. $J$. Colloid Interface Sci. 2019, 553, 350-363.

480 19. Bedrov, D.; Piquemal, J.-P.; Borodin, O.; MacKerell, A. D.; Roux, B.; Schröder, C., Molecular 481 Dynamics Simulations of Ionic Liquids and Electrolytes Using Polarizable Force Fields. Chem. Rev. 482 2019, $119(13), 7940-7995$. 
20. Goloviznina, K.; Canongia Lopes, J. N.; Costa Gomes, M.; Pádua, A. A. H., Transferable, Polarizable Force Field for Ionic Liquids. J. Chem. Theory Comput. 2019, 15 (11), 5858-5871. Theory and molecular dynamics simulation algorithm. J. Chem. Phys. 2003, 119 (6), 3025-3039. 22. Dequidt, A.; Devémy, J.; Pádua, A. A. H., Thermalized Drude Oscillators with the LAMMPS Molecular Dynamics Simulator. J. Chem. Inf. Model. 2016, 56 (1), 260-268.

23. Bernardes, C. E. S.; Shimizu, K.; Lopes, J. N. C.; Marquetand, P.; Heid, E.; Steinhauser, O.; Schröder, C., Additive polarizabilities in ionic liquids. Phys. Chem. Chem. Phys. 2016, 18 (3), 16651670 .

24. Heid, E.; Szabadi, A.; Schröder, C., Quantum mechanical determination of atomic polarizabilities of ionic liquids. Phys. Chem. Chem. Phys. 2018, 20 (16), 10992-10996.

25. Klajmon, M.; Červinka, C., Does Explicit Polarizability Improve Simulations of Phase Behavior of Ionic Liquids? J. Chem. Theory Comput. 2021, 17 (10), 6225-6239.

26. Canongia Lopes, J. N.; Padua, A. A. H., CL\&P: A generic and systematic force field for ionic liquids modeling. Theor. Chem. Acc. 2012, 131 (3), 1129.

27. Goloviznina, K.; Gong, Z.; Padua, A. A. H., The CL\&Pol polarizable force field for the simulation of ionic liquids and eutectic solvents. Wires Comput Mol Sci 2021.

500 28. Červinka, C.; Pádua, A. A. H.; Fulem, M., Thermodynamic Properties of Selected Homologous 501 Series of Ionic Liquids Calculated Using Molecular Dynamics. J. Phys. Chem. B 2016, 120 (9), 23622371.

29. Červinka, C.; Klajmon, M.; Štejfa, V., Cohesive Properties of Ionic Liquids Calculated from First Principles. Journal of Chemical Theory and Computation 2019, 15 (10), 5563-5578.

30. Plimpton, S.; Thompson, A.; Moore, S.; Kohlmayer, A., Large-scale Atomic/Molecular Massively Parallel Simulator. Large-scale Atomic/Molecular Massively Parallel Simulator 2018.

31. Plimpton, S., Fast Parallel Algorithms for Short-Range Molecular Dynamics. Journal of Computational Physics 1995, 117 (1), 1-19.

32. Martinez, L.; Andrade, R.; Birgin, E. G.; Martinez, J. M., PACKMOL: A Package for Building Initial Configurations for Molecular Dynamics Simulations. J Comput Chem 2009, 30 (13), 2157-2164. 33. Son, C. Y.; McDaniel, J. G.; Cui, Q.; Yethiraj, A., Proper Thermal Equilibration of Simulations with Drude Polarizable Models: Temperature-Grouped Dual-Nose-Hoover Thermostat. J Phys Chem Lett 2019, 10 (23), 7523-+.

34. Pádua, A. A. H.; Canongia Lopes, J. N. CL\&P: Molecular force field for ionic liquids (il.ff). https://github.com/agiliopadua/ilff/blob/master/il.ff (accessed Nov 27, 2020).

35. Pádua, A. A. H., Resolving dispersion and induction components for polarisable molecular simulations of ionic liquids. J. Chem. Phys. 2017, 146 (20), 204501.

36. Allen, M. P.; Tildesley, D. J., Computer simulation of liquids. Second edition. ed.; Oxford University Press: Oxford, United Kingdom, 2017; p xiv, 626 pages.

37. Navidi, W. C., Statistics for engineers and scientists. 3rd ed.; McGraw-Hill: New York, 2011; p xviii, $908 \mathrm{p}$.

38. Maginn, E. J.; Messerly, R. A.; Carlson, D. J.; Roe, D. R.; Elliot, J. R., Best Practices for Computing Transport Properties 1. Self-Diffusivity and Viscosity from Equilibrium Molecular Dynamics [Article v1.0]. LiveCoMS 2018, 1 (1), 6324.

39. Dong, Q.; Muzny, C. D.; Kazakov, A.; Diky, V.; Magee, J. W.; Widegren, J. A.; Chirico, R. D.; Marsh, K. N.; Frenkel, M., ILThermo: A free-access web database for thermodynamic properties of ionic liquids. J Chem Eng Data 2007, 52 (4), 1151-1159.

40. Tokuda, H.; Tsuzuki, S.; Susan, M. A. B. H.; Hayamizu, K.; Watanabe, M., How ionic are room-temperature ionic liquids? An indicator of the physicochemical properties. J Phys Chem B 2006, 110 (39), 19593-19600. 
531 41. Domanska, U.; Krolikowski, M., Phase equilibria study of the binary systems (1-butyl-3532 methylimidazolium tosylate ionic liquid plus water, or organic solvent). J Chem Thermodyn 2010, 42 533 (3), 355-362.

534 42. Kocian, Š.; Štejfa, V., Personal communication.

535 43. Dequidt, A. Diffraction code, Université Clermont Auvergne, 2016.

536 44. Research, N. C. f. N. Neutron Scattering Lengths and Cross Sections of the Elements and Their 537 Isotopes. https://www.ncnr.nist.gov/resources/n-lengths/list.html (accessed Dec 15, 2021).

538 45. Mao, X.; Brown, P.; Červinka, C.; Hazell, G.; Li, H.; Ren, Y.; Chen, D.; Atkin, R.; Eastoe, J.; 539 Grillo, I.; Padua, A. A. H.; Costa Gomes, M. F.; Hatton, T. A., Self-assembled nanostructures in ionic 540 liquids facilitate charge storage at electrified interfaces. Nature Mater. 2019, 18 (12), 1350-1357. 
543 Table of contents

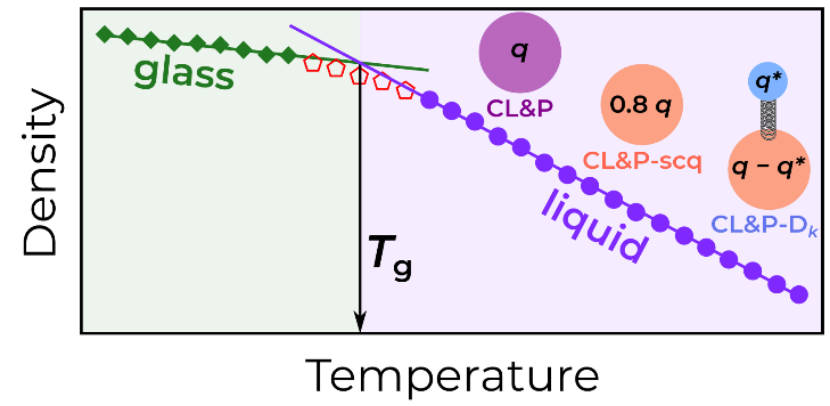

545

546 


\title{
Does Explicit Polarizability Improve Molecular-Dynamics Predictions of Glass Transition Temperatures of Ionic Liquids?
}

\author{
Martin Klajmon and Ctirad Červinka* \\ Department of Physical Chemistry, Faculty of Chemical Engineering, University of Chemistry and \\ Technology, Prague, Technická 5, 16628 Prague 6, Czech Republic
}

${ }^{*}$ Corresponding author: cervinkc@vscht.cz

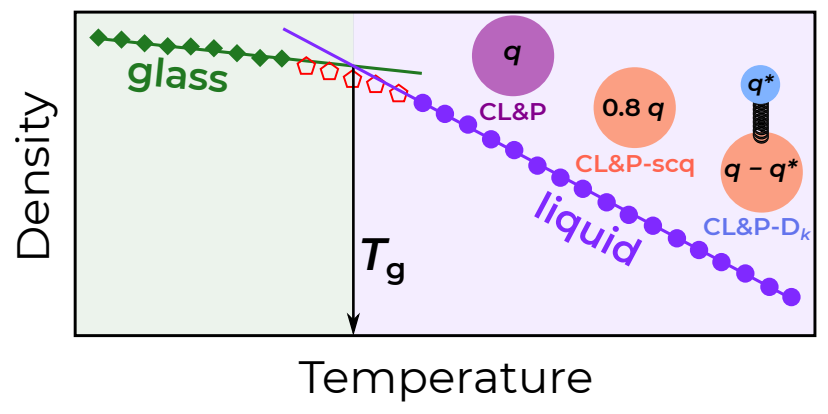



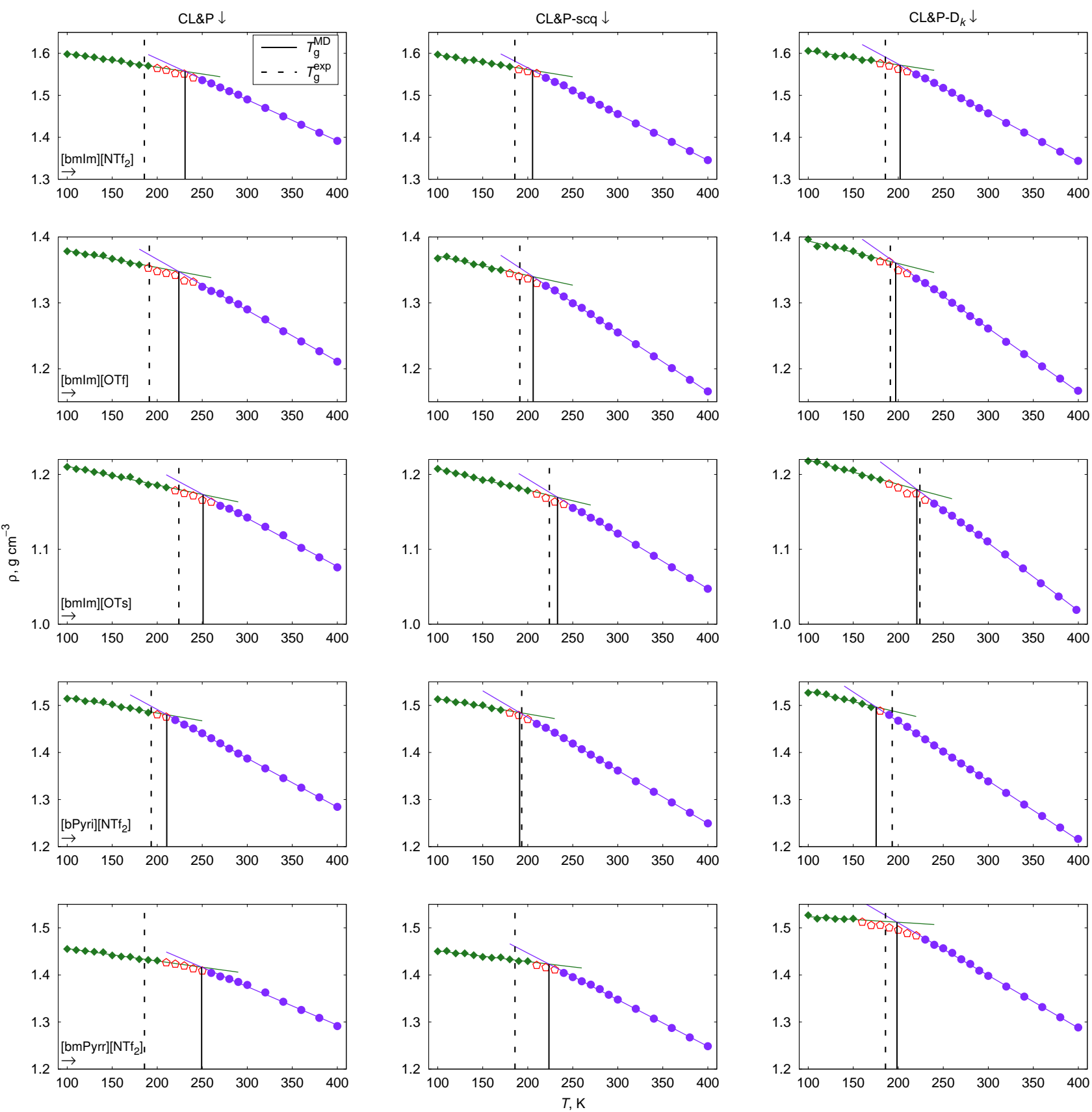

Figure S1: Determination of the glass transition temperatures $\left(T_{\mathrm{g}}\right)$ of the considered ionic liquids by the trend shift method from the temperature-dependent densities simulated by the three different force field models. Green and purple points were used for linear extrapolations of the trends of the glassy and liquid phases, respectively. Data points excluded from the $T_{\mathrm{g}}$ evaluation are displayed with empty points. 

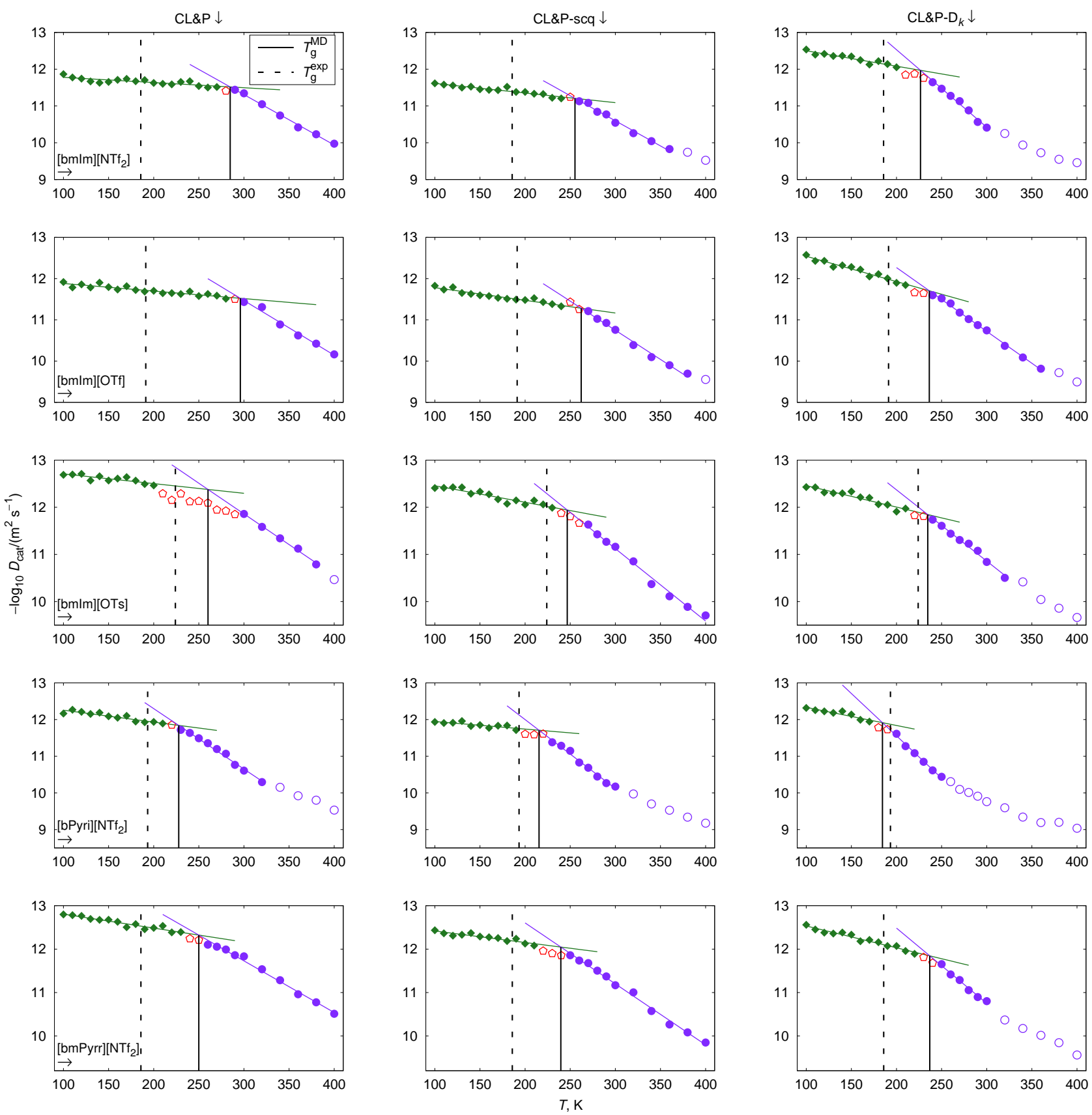

Figure S2: Determination of the glass transition temperatures $\left(T_{\mathrm{g}}\right)$ of the considered ionic liquids by the trend shift method from the temperature-dependent cation diffusivities simulated by the three different force field models. Green and purple points were used for linear extrapolations of the trends of the glassy and liquid phases, respectively. Data points excluded from the $T_{\mathrm{g}}$ evaluation are displayed with empty points. 

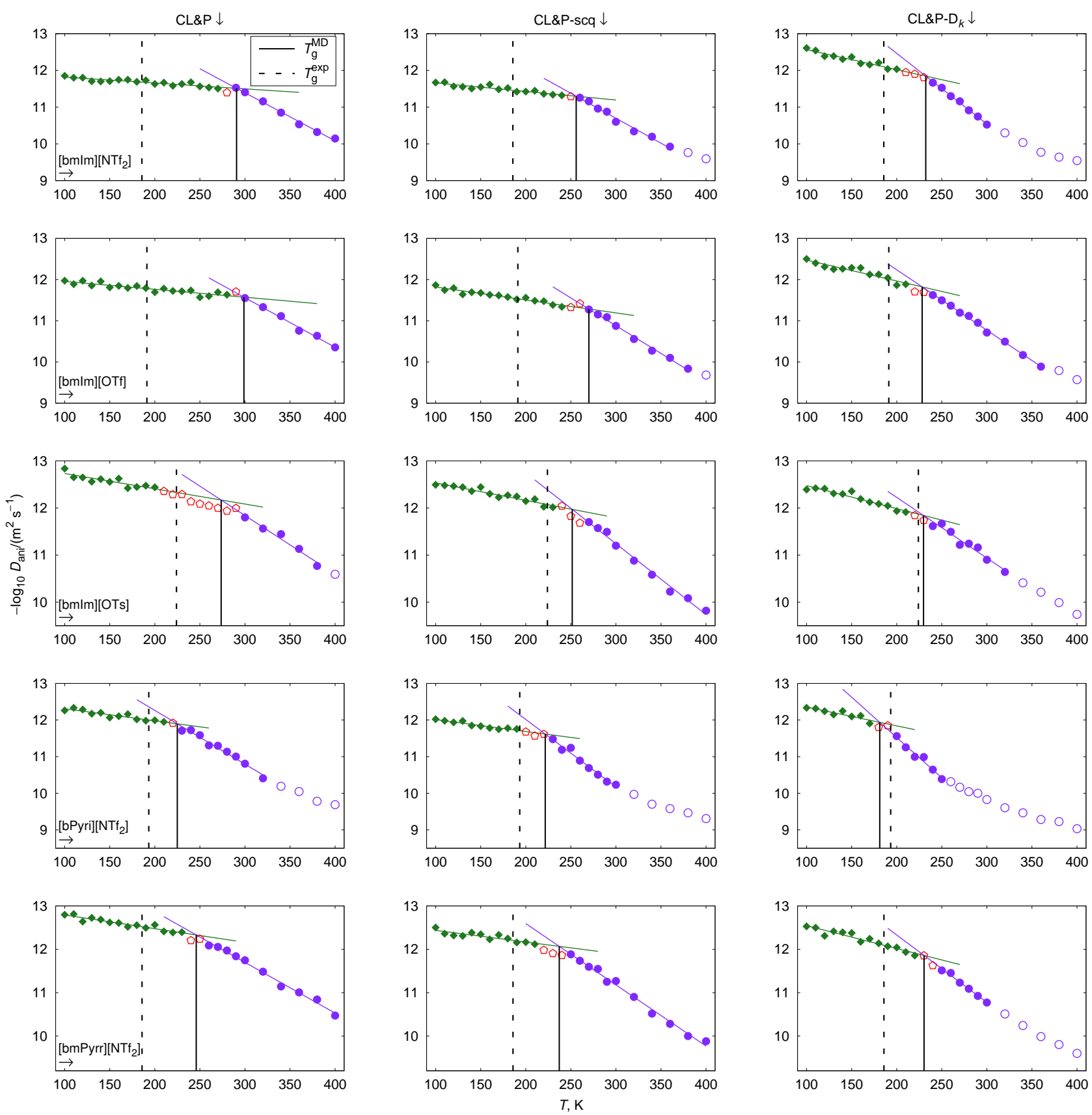

Figure S3: Determination of the glass transition temperatures $\left(T_{\mathrm{g}}\right)$ of the considered ionic liquids by the trend shift method from the temperature-dependent anion diffusivities. The symbols have the same meaning as in Figure S2. 

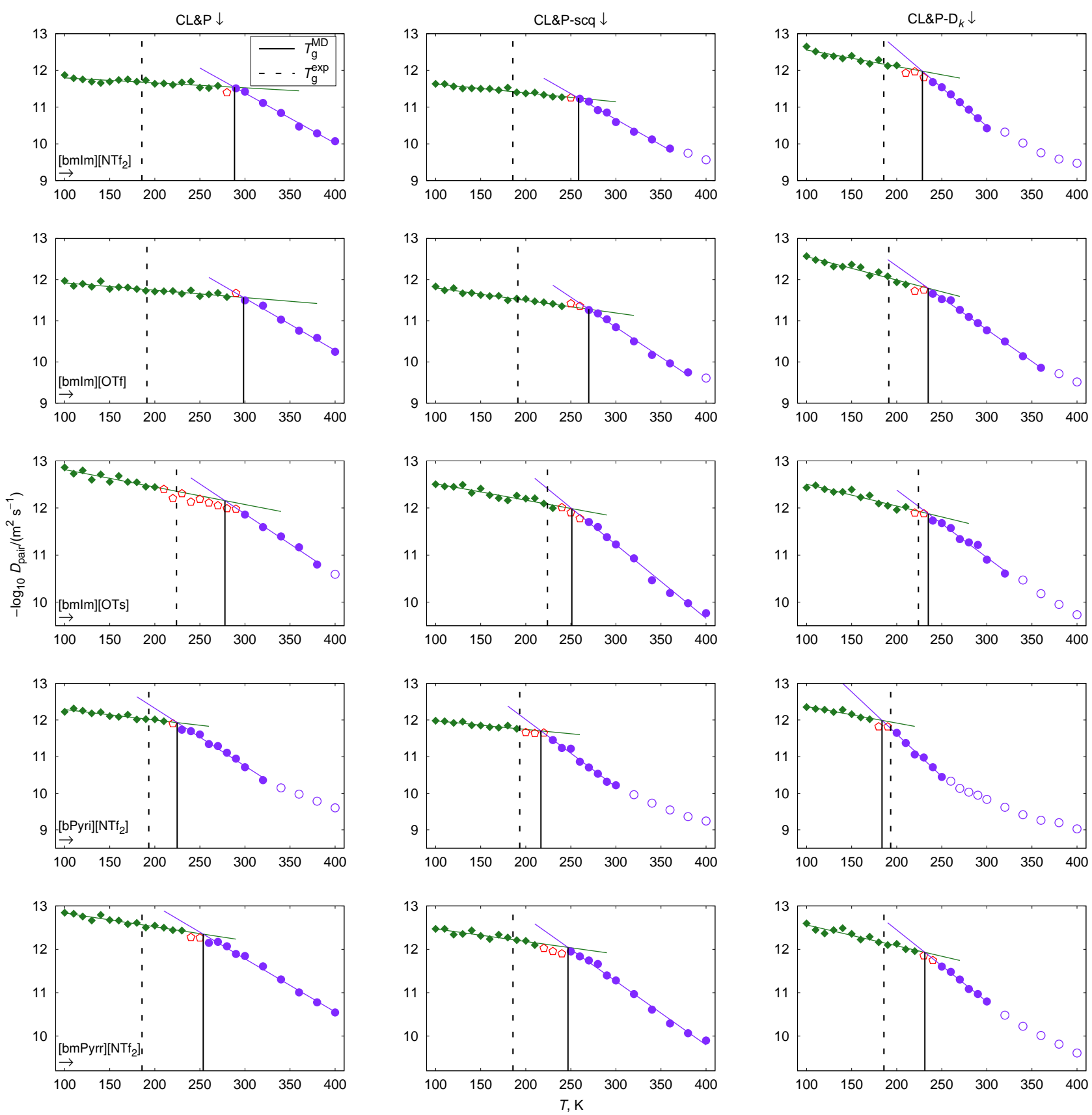

Figure S4: Determination of the glass transition temperatures $\left(T_{\mathrm{g}}\right)$ of the considered ionic liquids by the trend shift method from the temperature-dependent ion-pair diffusivities. The symbols have the same meaning as in Figure S2. 


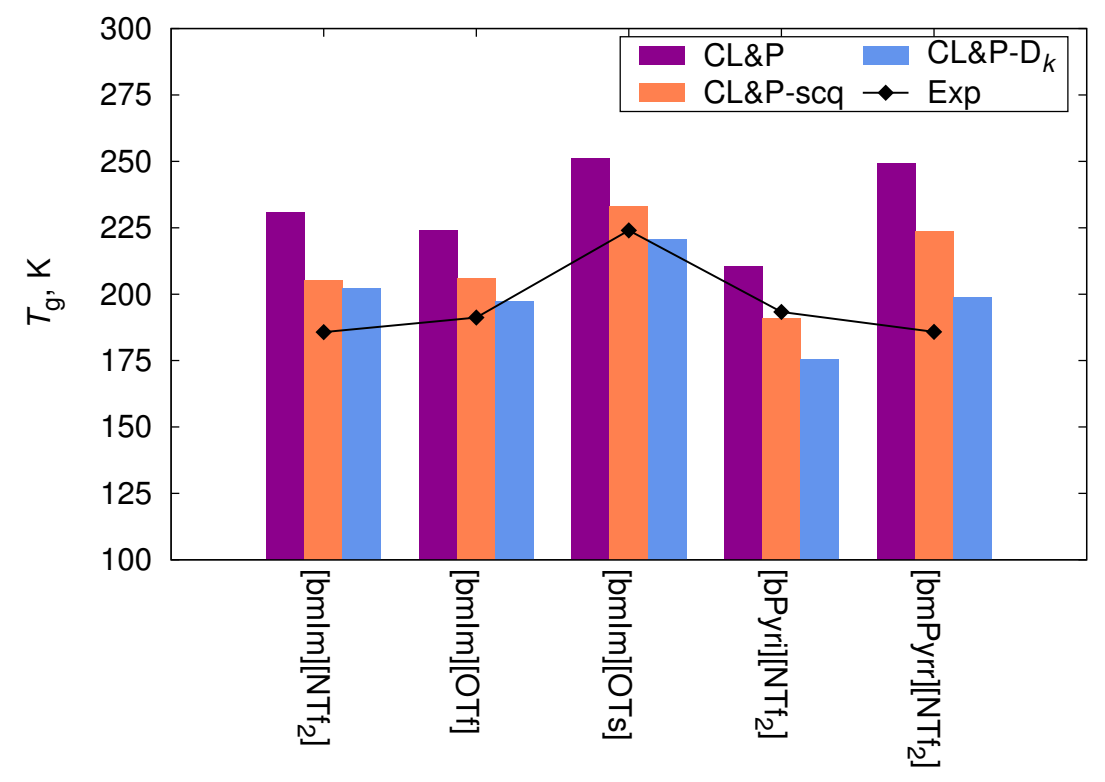

Figure S5: Comparison of experimental $T_{\mathrm{g}}$ values and those calculated in this work from densities $\left(T_{\mathrm{g}}^{\rho}\right)$ simulated using the various force field models for all 5 ILs.

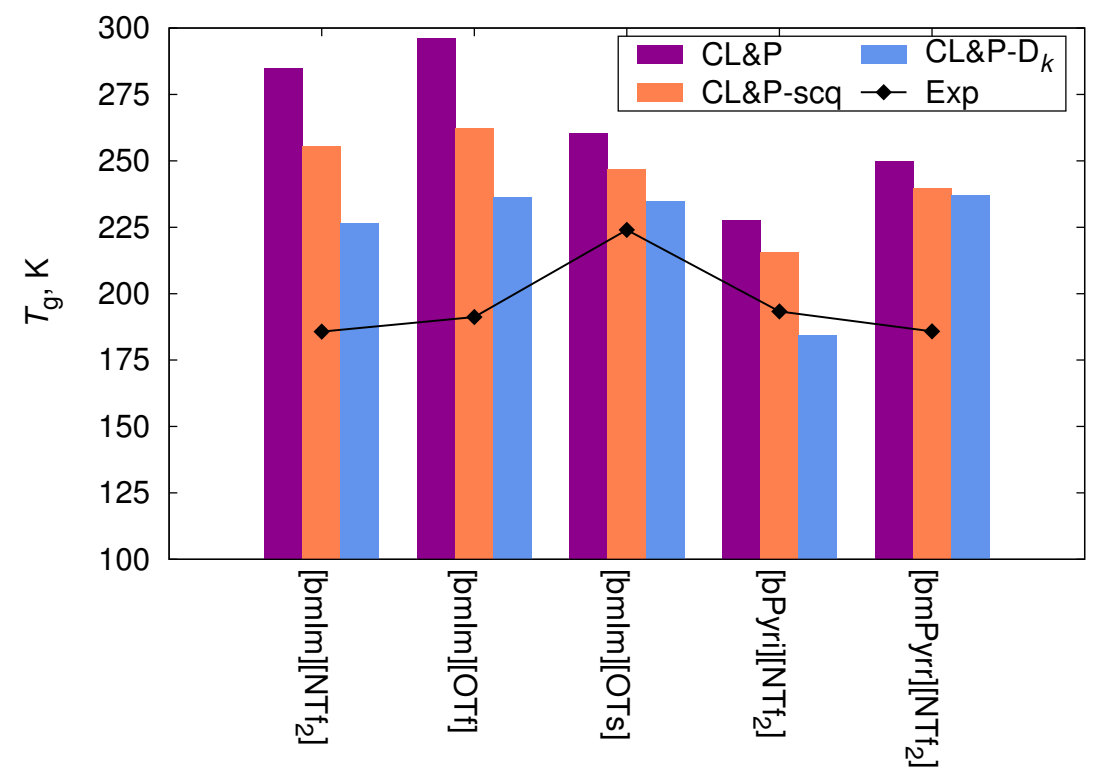

Figure S6: Comparison of experimental $T_{\mathrm{g}}$ values and those calculated in this work from cation diffusivities $\left(T_{\mathrm{g}}^{D}\right)$ simulated using the various force field models for all 5 ILs. 

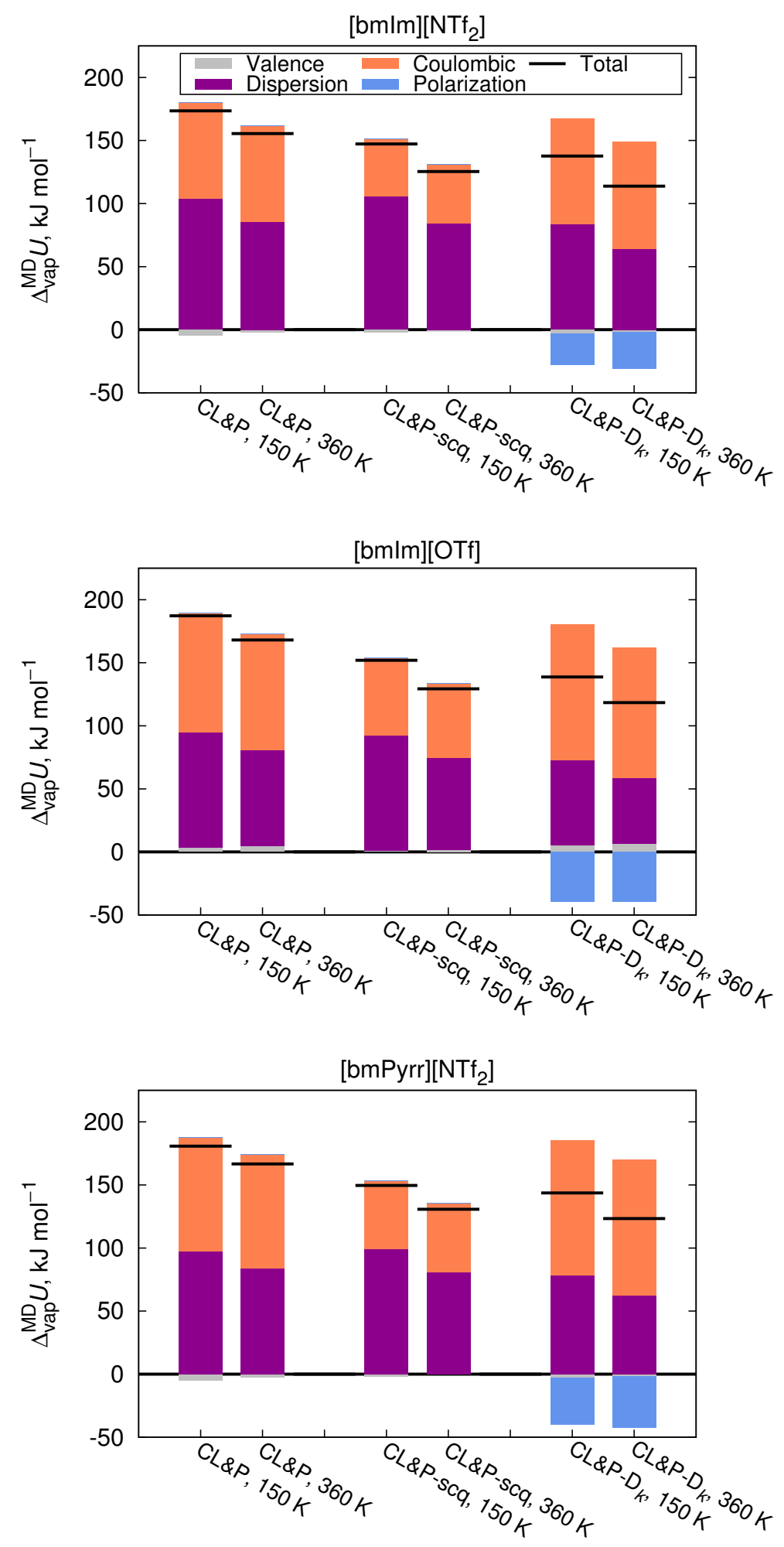

Figure S7: Vaporization energies and their individual mechanistic components calculated for $[\mathrm{bmIm}]\left[\mathrm{NTf}_{2}\right],[\mathrm{bmIm}][\mathrm{OTf}]$, and [bmPyrr] $\left[\mathrm{NTf}_{2}\right]$ using the various force field models. 

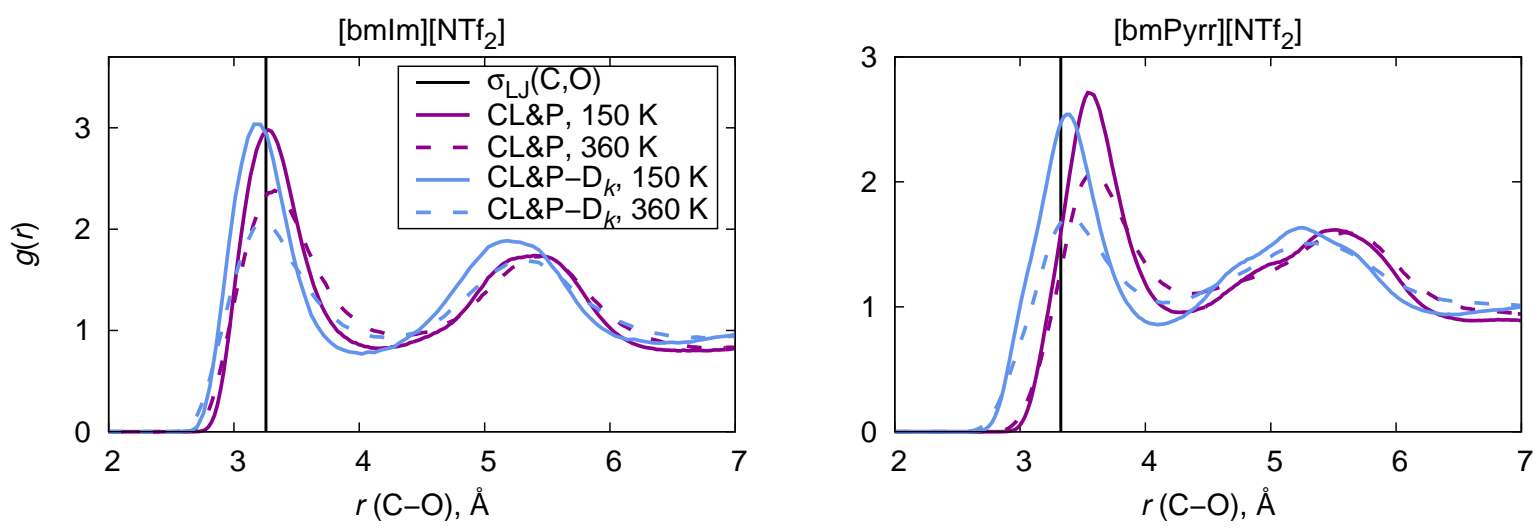

Figure S8: Radial distribution functions of $\mathrm{C}-\mathrm{O}$ calculated for $[\mathrm{bmIm}]\left[\mathrm{NTf}_{2}\right]$ and $[\mathrm{bmPyrr}]\left[\mathrm{NTf}_{2}\right]$ using the $\mathrm{CL} \& \mathrm{P}$ and $\mathrm{CL} \& \mathrm{P}-\mathrm{D}_{k}$ force field models at two different temperatures. For [bmIm], C represents the $\mathrm{C}_{2}$ atom of the imidazolium ring, whereas it corresponds to the $\mathrm{C}_{2}$ and $\mathrm{C}_{5}$ atoms of the pyrrolidinium ring in [bmPyrr]. O represents the oxygen atoms of $\left[\mathrm{NTf}_{2}\right]$.

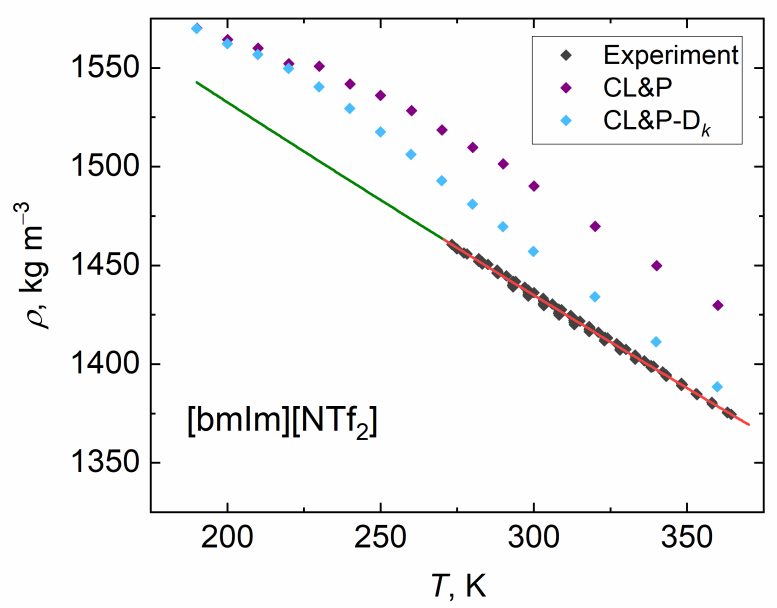

Figure S9: Comparison of calculated and experimental [1-9] densities of liquid [bmIm] $\left.\mathrm{NTf}_{2}\right]$.

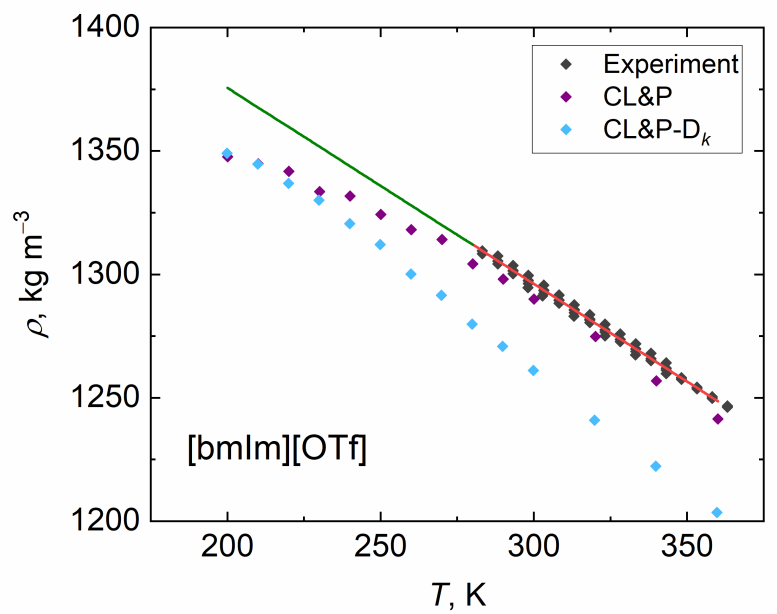

Figure S10: Comparison of calculated and experimental [10-15] densities of liquid [bmIm][OTf]. 


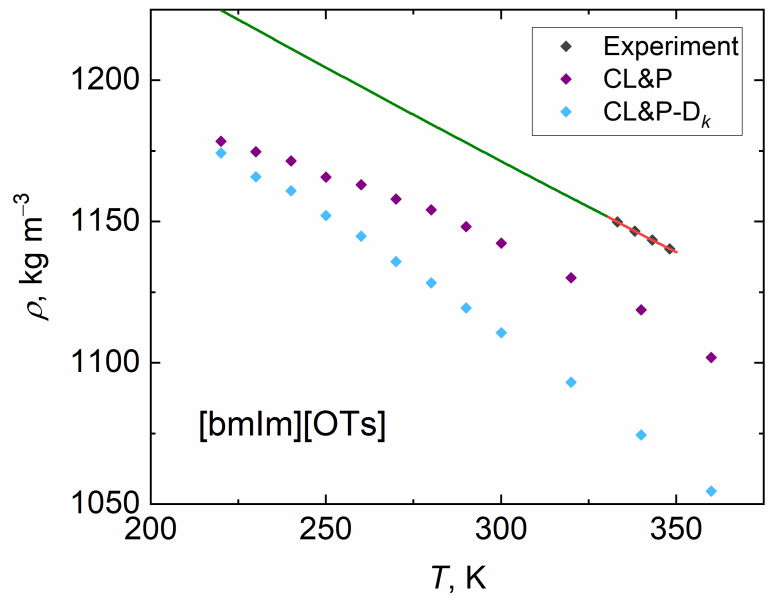

Figure S11: Comparison of calculated and experimental [16] densities of liquid [bmIm] [OTs].

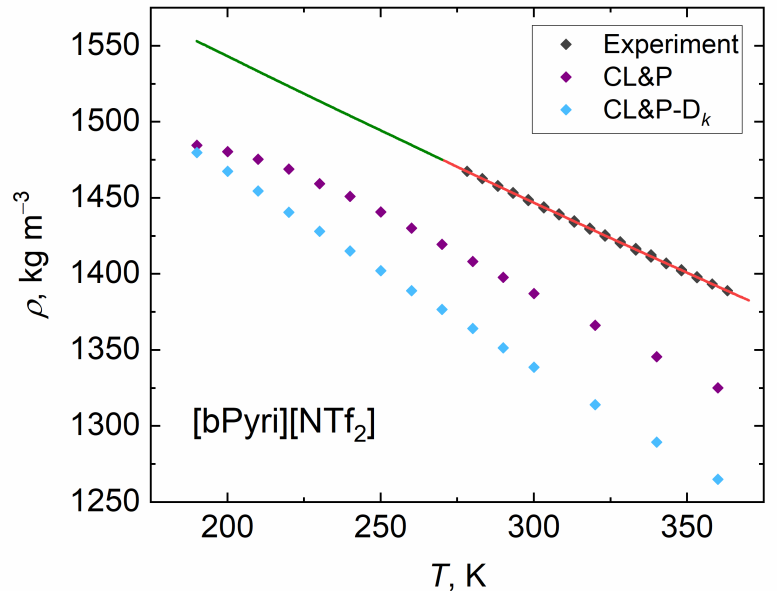

Figure S12: Comparison of calculated and experimental [17-21] densities of liquid [bPyri][ $\left.\mathrm{NTf}_{2}\right]$.

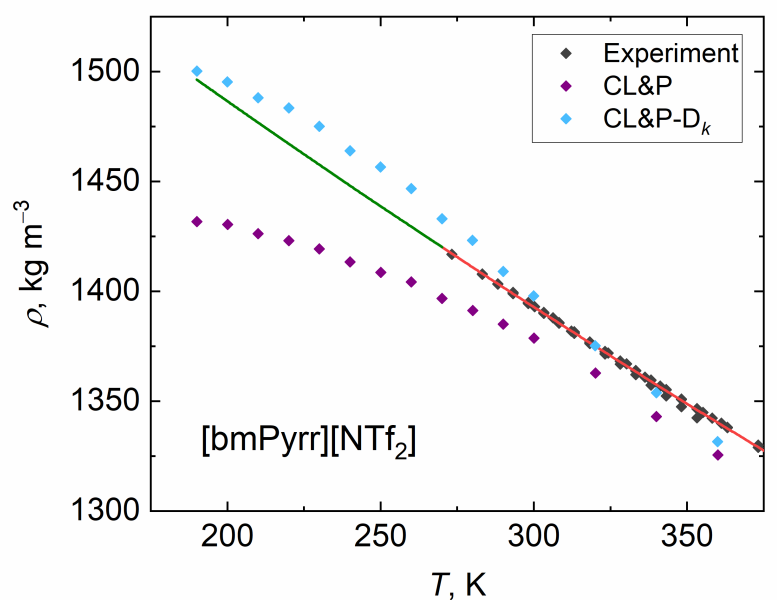

Figure S13: Comparison of calculated and experimental $[10,11,22-27]$ densities of liquid $\left[\right.$ bmPyrr] $\left[\mathrm{NTf}_{2}\right]$. 


\section{References}

[1] Almeida, H. F. D.; Canongia Lopes, J. N.; Rebelo, L. P. N.; Coutinho, J. A. P.; Freire, M. G.; Marrucho, I. M. Densities and Viscosities of Mixtures of Two Ionic Liquids Containing a Common Cation. 2016, 61, 2828-2843.

[2] Zorębski, M.; Zorębski, E.; Dzida, M.; Skowronek, J.; Jężak, S.; Goodrich, P.; Jacquemin, J. Ultrasonic Relaxation Study of 1-Alkyl-3-methylimidazolium-Based Room-Temperature Ionic Liquids: Probing the Role of Alkyl Chain Length in the Cation. 2016, 120, 3569-3581.

[3] Widowati, E.; Lee, M.-J. P-V-T properties of binary mixtures of the ionic liquid 1-butyl-3methylimidazolium bis(trifluoromethylsulfonyl)imide with anisole or acetophenone at elevated pressures. 2013, 63, 95-101.

[4] Součková, M.; Klomfar, J.; Pátek, J. Measurements and group contribution analysis of $0.1 \mathrm{MPa}$ densities for still poorly studied ionic liquids with the [PF6] and [NTf2] anions. 2014, 7r, 31-39.

[5] Khalil, R.; Chaabene, N.; Azar, M.; Malham, I. B.; Turmine, M. Effect of the chain lengthening on transport properties of imidazolium-based ionic liquids. 2020, 503, 112316.

[6] Kanakubo, M.; Harris, K. R. Density of 1-Butyl-3-methylimidazolium Bis(trifluoromethanesulfonyl)amide and 1-Hexyl-3-methylimidazolium Bis(trifluoromethanesulfonyl)amide over an Extended Pressure Range up to $250 \mathrm{MPa}$. 2015, 60, 1408-1418.

[7] Jacquemin, J.; Husson, P.; Majer, V.; Costa Gomes, M. F. Influence of the Cation on the Solubility of $\mathrm{CO} 2$ and $\mathrm{H} 2$ in Ionic Liquids Based on the Bis(trifluoromethylsulfonyl)imide Anion. 2007, 36, 967-979.

[8] Gomes de Azevedo, R.; Esperança, J. M. S. S.; Szydlowski, J.; Visak, Z. P.; Pires, P. F.; Guedes, H. J. R.; Rebelo, L. P. N. Thermophysical and thermodynamic properties of ionic liquids over an extended pressure range: [bmim] [NTf2] and [hmim][NTf2]. 2005, 37, 888-899.

[9] Geppert-Rybczyńska, M.; Sitarek, M. Acoustic and Volumetric Properties of Binary Mixtures of Ionic Liquid 1-Butyl-3-methylimidazolium Bis(trifluoromethylsulfonyl)imide with Acetonitrile and Tetrahydrofuran. 2014, 59, 1213-1224.

[10] Seoane, R. G.; Corderí, S.; Gómez, E.; Calvar, N.; González, E. J.; Macedo, E. A.; Domínguez, n. Temperature Dependence and Structural Influence on the Thermophysical Properties of Eleven Commercial Ionic Liquids. 2012, 51, 2492-2504.

[11] Musiał, M.; Zorębski, E.; Zorębski, M.; Dzida, M. Effect of alkyl chain length in cation on thermophysical properties of two homologous series: 1-alkyl-1-methylpyrrolidinium bis(trifluoromethylsulfonyl)imides and 1-alkyl-3-methylimidazolium trifluoromethanesulfonates. 2019, 293, 111511.

[12] Mbondo Tsamba, B. E.; Sarraute, S.; Traikia, M.; Husson, P. Transport Properties and Ionic Association in Pure Imidazolium-Based Ionic Liquids as a Function of Temperature. 2014, 59, $1747-1754$.

[13] González, E. J.; Domínguez, n.; Macedo, E. A. Physical and Excess Properties of Eight Binary Mixtures Containing Water and Ionic Liquids. 2012, 57, 2165-2176.

[14] González, E. J.; Calvar, N.; Domínguez, n.; Macedo, E. A. Osmotic and apparent molar properties of binary mixtures alcohol+1-butyl-3-methylimidazolium trifluoromethanesulfonate ionic liquid. 2013, 61, 64-73.

[15] García-Miaja, G.; Troncoso, J.; Romaní, L. Excess properties for binary systems ionic liquid+ethanol: Experimental results and theoretical description using the ERAS model. 2008, $274,59-67$.

[16] Domańska, U.; Królikowski, M. Phase equilibria study of the binary systems (1-butyl-3methylimidazolium tosylate ionic liquid+water, or organic solvent). 2010, 42, 355-362.

[17] Zeng, S.; Wang, J.; Bai, L.; Wang, B.; Gao, H.; Shang, D.; Zhang, X.; Zhang, S. Highly Selective Capture of $\mathrm{CO} 2$ by Ether-Functionalized Pyridinium Ionic Liquids with Low Viscosity. 2015, 29, 6039-6048. 
[18] Santos, D.; Santos, M.; Franceschi, E.; Dariva, C.; Barison, A.; Mattedi, S. Experimental Density of Ionic Liquids and Thermodynamic Modeling with Group Contribution Equation of State Based on the Lattice Fluid Theory. 2016, 61, 348-353.

[19] Oliveira, F. S.; Freire, M. G.; Carvalho, P. J.; Coutinho, J. A. P.; Lopes, J. N. C.; Rebelo, L. P. N.; Marrucho, I. M. Structural and Positional Isomerism Influence in the Physical Properties of Pyridinium NTf2-Based Ionic Liquids: Pure and Water-Saturated Mixtures. 2010, 55, 4514-4520.

[20] Larriba, M.; García, S.; Navarro, P.; García, J.; Rodríguez, F. Physical Properties of NButylpyridinium Tetrafluoroborate and N-Butylpyridinium Bis(trifluoromethylsulfonyl)imide Binary Ionic Liquid Mixtures. 2012, 57, 1318-1325.

[21] Bounsiar, R.; Gascón, I.; Amireche, F.; Lafuente, C. Volumetric properties of three pyridiniumbased ionic liquids with a common cation or anion. 2020, 521, 112732.

[22] Geppert-Rybczyńska, M.; Lehmann, J. K.; Heintz, A. Physicochemical properties of two 1-alkyl1-methylpyrrolidinium bis[(trifluoromethyl)sulfonyl]imide ionic liquids and of binary mixtures of 1-butyl-1-methylpyrrolidinium bis[(trifluoromethyl)sulfonyl]imide with methanol or acetonitrile. 2014, 71, 171-181.

[23] Harris, K. R.; Woolf, L. A.; Kanakubo, M.; Rüther, T. Transport Properties of N-Butyl-Nmethylpyrrolidinium Bis(trifluoromethylsulfonyl)amide. 2011, 56, 4672-4685.

[24] Sánchez-Ramírez, N.; Assresahegn, B. D.; Bélanger, D.; Torresi, R. M. A Comparison among Viscosity, Density, Conductivity, and Electrochemical Windows of N-n-Butyl-N-methylpyrrolidinium and Triethyl-n-pentylphosphonium Bis(fluorosulfonyl imide) Ionic Liquids and Their Analogues Containing Bis(trifluoromethylsulfonyl) Imide Anion. 2017, 62, 3437-3444.

[25] Sas, O. G.; Ivaniš, G. R.; Kijevčanin, M. L.; González, B.; Domínguez, A.; Radović, I. R. Densities and Derived Volumetric Properties of Ionic Liquids with [Nf2] and [NTf2] Anions at High Pressures. 2018, 63, 954-964.

[26] Vraneš, M.; Dožić, S.; Djeric, V.; Gadžurić, S. Physicochemical Characterization of 1-Butyl-3methylimidazolium and 1-Butyl-1-methylpyrrolidinium Bis(trifluoromethylsulfonyl)imide. 2012, 57, 1072-1077.

[27] Vraneš, M.; Tot, A.; Papović, S.; Zec, N.; Dožić, S.; Gadžurić, S. Ideal and non-ideal behaviour of 1-butyl-1-methylpyrrolydinium bis(trifluoromethylsulfonyl)imide $+\gamma$-butyrolactone binary mixtures. 2015, 81, 66-76. 